NATIONAL LABORATORY

\title{
Laboratory Guide for Residual Stress Sample Alignment and Experiment Planning-October 2011 Version
}

Prepared by:

Paris Cornwell

Jeff Bunn

Josh Schmidlin

Camden Hubbard

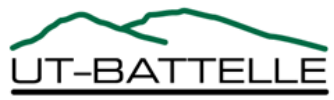




\section{DOCUMENT AVAILABILITY}

Reports produced after January 1, 1996, are generally available free via the U.S. Department of Energy (DOE) Information Bridge.

Web site http://www.osti.gov/bridge

Reports produced before January 1, 1996, may be purchased by members of the public from the following source.

National Technical Information Service

5285 Port Royal Road

Springfield, VA 22161

Telephone 703-605-6000 (1-800-553-6847)

TDD 703-487-4639

Fax 703-605-6900

E-mail info@ntis.gov

Web site http://www.ntis.gov/support/ordernowabout.htm

Reports are available to DOE employees, DOE contractors, Energy Technology Data Exchange (ETDE) representatives, and International Nuclear Information System (INIS) representatives from the following source.

Office of Scientific and Technical Information

P.O. Box 62

Oak Ridge, TN 37831

Telephone 865-576-8401

Fax 865-576-5728

E-mail reports@osti.gov

Web site http://www.osti.gov/contact.html

This report was prepared as an account of work sponsored by an agency of the United States Government. Neither the United States Government nor any agency thereof, nor any of their employees, makes any warranty, express or implied, or assumes any legal liability or responsibility for the accuracy, completeness, or usefulness of any information, apparatus, product, or process disclosed, or represents that its use would not infringe privately owned rights. Reference herein to any specific commercial product, process, or service by trade name, trademark, manufacturer, or otherwise, does not necessarily constitute or imply its endorsement, recommendation, or favoring by the United States Government or any agency thereof. The views and opinions of authors expressed herein do not necessarily state or reflect those of the United States Government or any agency thereof. 
Materials Science and Technology Division

\title{
Laboratory Guide for Residual Stress Sample Alignment and Experiment Planning
}

Paris Cornwell, Jeff Bunn, Josh Schmidlin, Camden Hubbard

Date Published: April 2012

\author{
Prepared by \\ OAK RIDGE NATIONAL LABORATORY \\ Oak Ridge, Tennessee 37831-6283 \\ managed by \\ UT-BATTELLE, LLC \\ for the \\ U.S. DEPARTMENT OF ENERGY \\ under contract DE-AC05-00OR22725
}




\section{Contents}

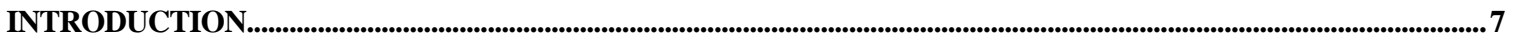

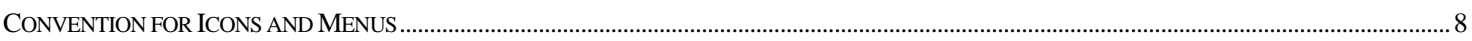

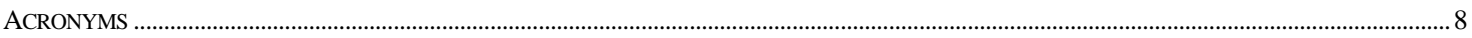

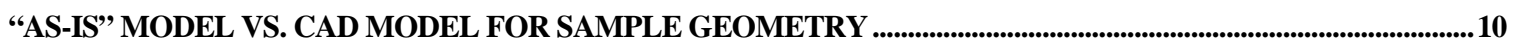

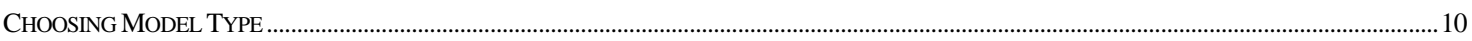

CONSIDERATIONS FOR ESTABLISHING THE SAMPLE COORDINATE SYSTEM (SCS) AND DEFINING

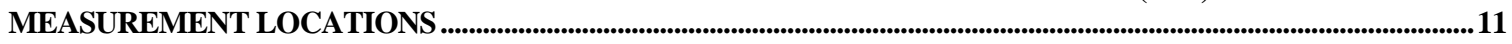

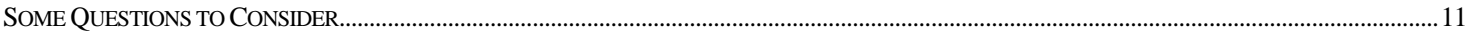

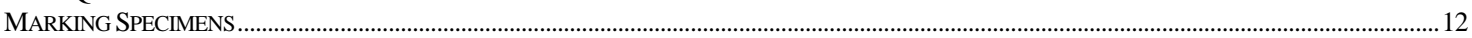

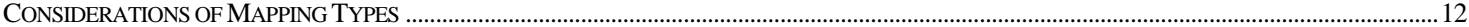

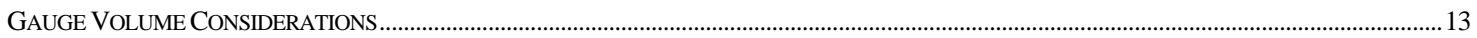

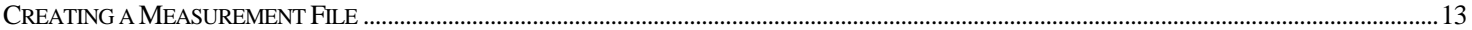

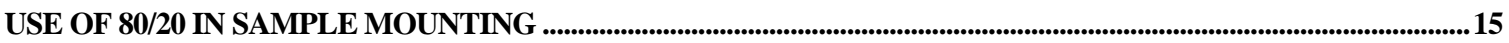

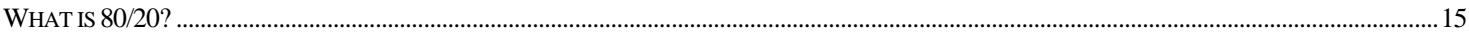

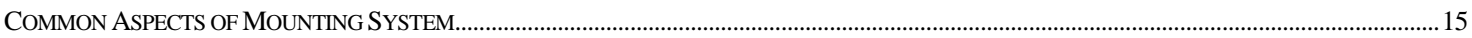

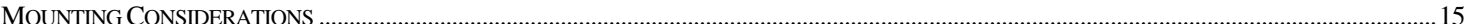

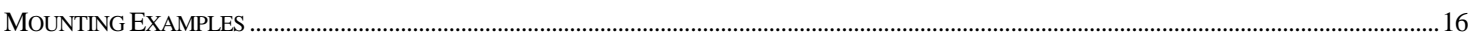

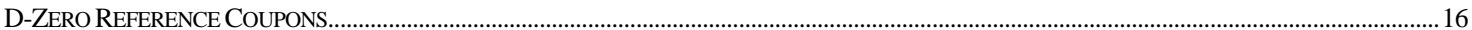

MOUNTING SAMPLE ON INDEXER TABLE AND ADDING FIDUCIAL POINTS..................................................17

SAMPLE MOUNTING CONSIDERATIONS FOR SCAN ARM MEASUREMENT …………………………………………..........................................

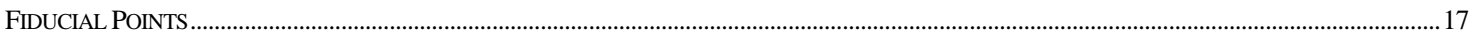

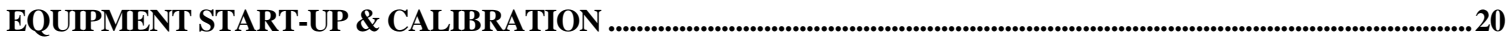

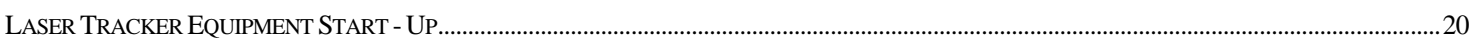

SCANARM EQUIPMENT START - UP _...

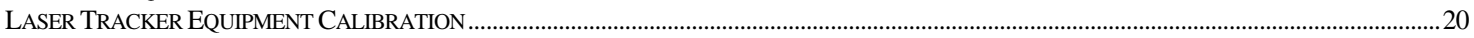

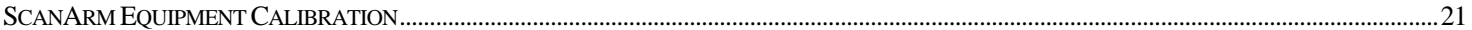

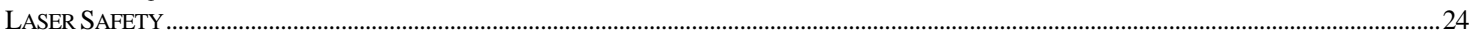

TRANSFORMING FROM WORLD TO THE SAMPLE COORDINATE SYSTEM (SCS) WITH

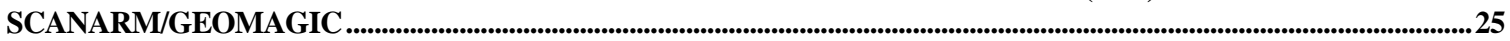

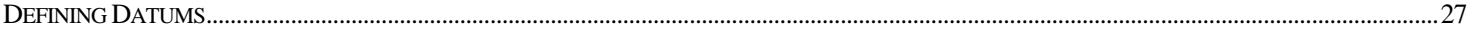

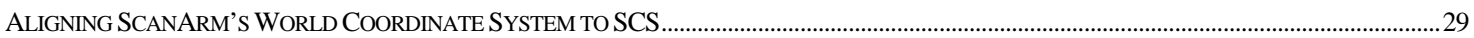

TRANSFORMING FROM WORLD TO THE SAMPLE COORDINATE SYSTEM (SCS) WITH FAROLASER

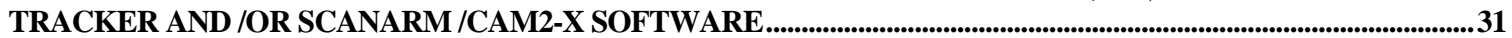

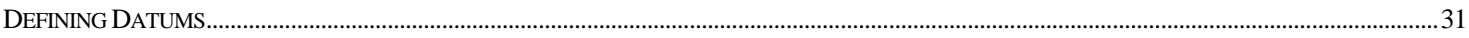

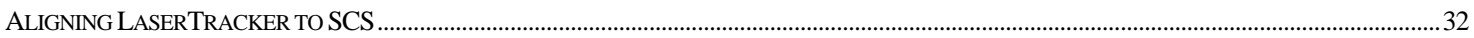

MEASURING THE FIDUCIAL POINTS IN THE SCS ............................................................................................................3.

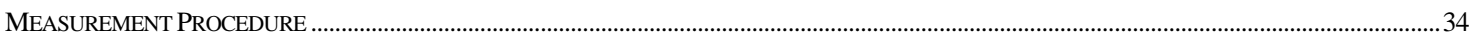

OBTAINING “AS-IS” SURFACE MODEL FOR THE SAMPLE ...........................................................................................38

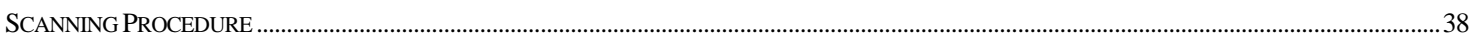

REFINING, DECIMATING AND EXPORTING THE SURFACE MODEL .........................................................42

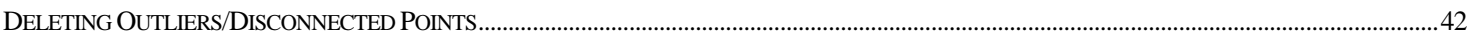

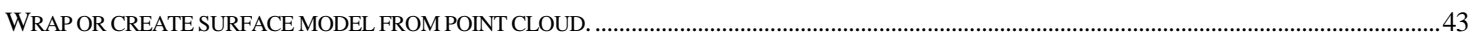

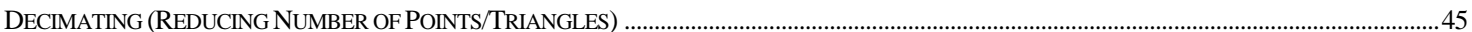

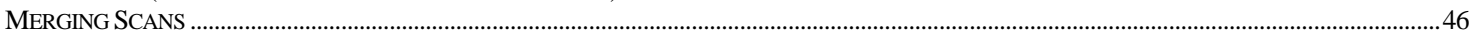

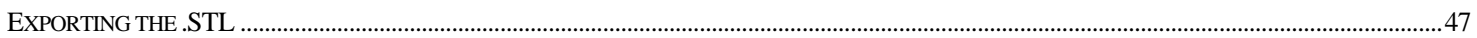

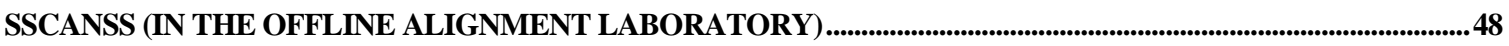

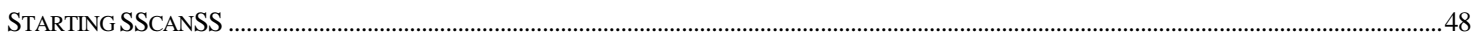




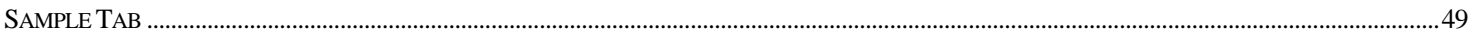

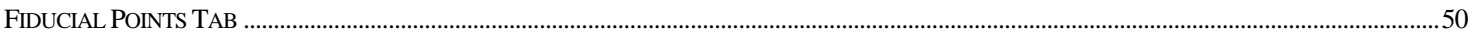

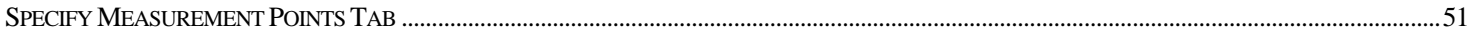

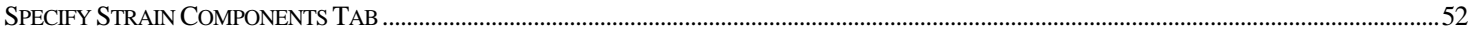

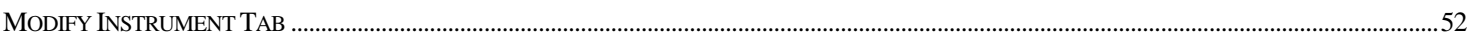

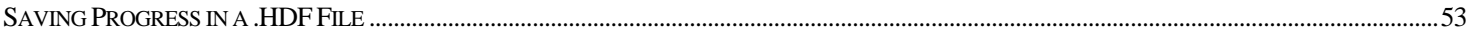

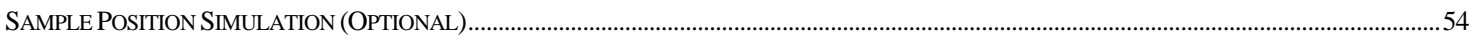

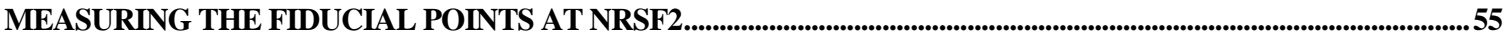

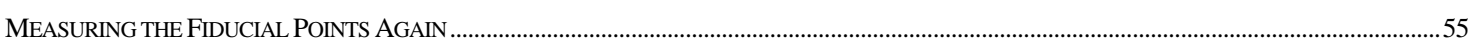

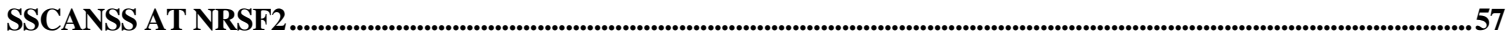

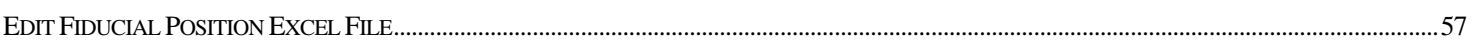

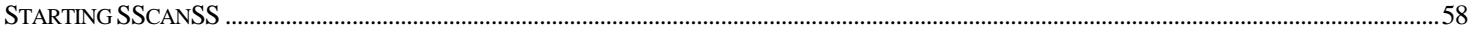

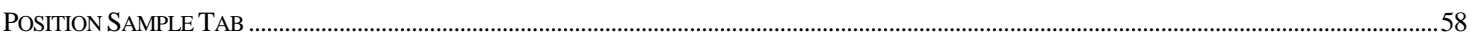

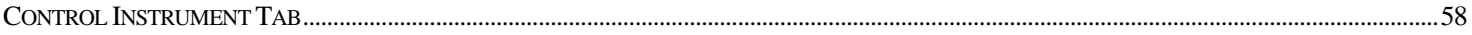

MEASURING THE FIDUCIAL POINTS AT VULCAN.............................................................................................................59

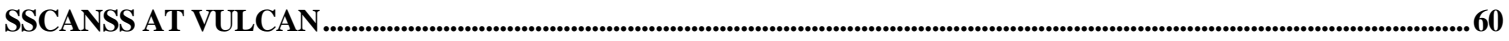

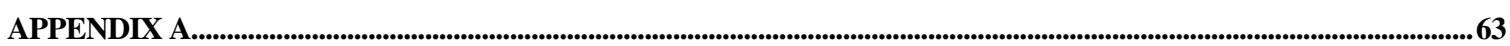

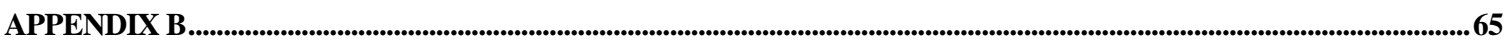




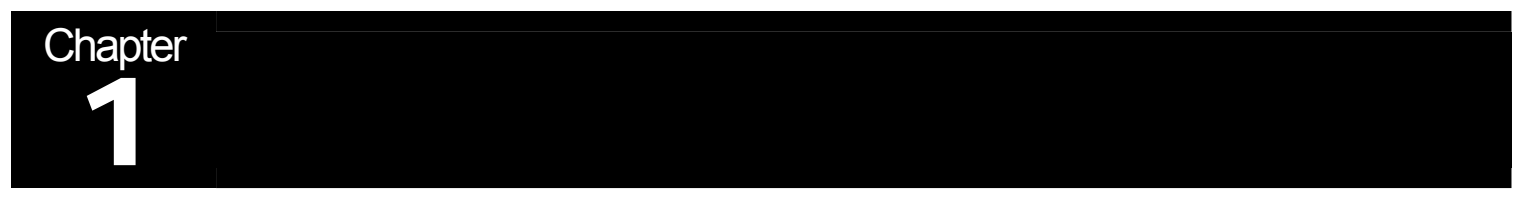

\section{Introduction}

The December 2010 version of the guide, ORNL/TM-2008/159, by Jeff Bunn, Josh Schmidlin, Camden Hubbard, and Paris Cornwell, has been further revised due to a major change in the GeoMagic Studio software for constructing a surface model. The Studio software update also includes a plug-in module to operate the FARO Scan Arm. Other revisions for clarity were also made.

The purpose of this revision document is to guide the reader through the process of laser alignment used by NRSF2 at HFIR and VULCAN at SNS ${ }^{1}$. This system was created to increase the spatial accuracy of the measurement points in a sample, reduce the use of neutron time used for alignment, improve experiment planning, and reduce operator error. The need for spatial resolution has been driven by the reduction in gauge volumes to the sub-millimeter level, steep strain gradients in some samples, and requests to mount multiple samples within a few days for relating data from each sample to a common sample coordinate system.

The first step in this process involves mounting the sample on an indexer table in a laboratory set up for offline sample mounting and alignment in the same manner it would be mounted at either instrument. In the shared laboratory, a FARO ScanArm is used to measure the coordinates of points on the sample surface ("point cloud"), specific features and fiducial points. A Sample Coordinate System (SCS) needs to be established first. This is an advantage of the technique because the SCS can be defined in such a way to facilitate simple definition of measurement points within the sample.

Next, samples are typically mounted to a frame of $80 / 20^{2}$ and fiducial points are attached to the sample or frame then measured in the established sample coordinate system. The laser scan probe on the ScanArm can then be used to scan in an "as-is" model of the sample as well as mounting hardware. GeoMagic Studio ${ }^{3} 12$ is the software package used to construct the model from the point cloud the scan arm creates. Once a model, fiducial, and measurement files are created, a special program, called SScanSS $S^{4,5}$ combines the information and by simulation of the sample on the diffractometer can help plan the experiment before using neutron time.

Finally, the sample is mounted on the relevant stress measurement instrument and the fiducial points are measured again. In the HFIR beam room, a laser tracker is used in conjunction with a program called CAM2 to measure the fiducial points in the NRSF2 instrument's sample positioner coordinate system. SScanSS is then used again to perform a coordinate system transformation of the measurement file locations to the sample positioner coordinate system. A procedure file is then written with the coordinates in the sample positioner coordinate system for the desired measurement locations. This file is often called a script or command file and can be further modified using excel. 
It is very important to note that this process is not a linear one, but rather, it often is iterative. Many of the steps in this guide are interdependent on one another. It is very important to discuss the process as it pertains to the specific sample being measured. What works with one sample may not necessarily work for another. This guide attempts to provide a typical work flow that has been successful in most cases.

\section{Convention for Icons and Menus}

In this guide, a convention was chosen to differentiate between icons, menus, pull down menus and checkboxes. The conventions as followed in the guide are listed below

$$
\begin{aligned}
& \text { Italics - checkboxes } \\
& \text { Bold - Icon } \\
& \text { Pull down Menu - "quotations" } \\
& \text { Pull-down menu (in SScanSS) - "Bold with quotations" } \\
& \text { Important information - Underline or Bold Underline }
\end{aligned}
$$

\section{Acronyms}

HFIR - High Flux Isotope Reactor

NRSF2 $-2^{\text {nd }}$ Generation Neutron Residual Stress Mapping Facility at HFIR

SCS - Sample Coordinate System

SMR - Spherically Mounted Retroreflector

SNS - Spallation Neutron Source

SPS - Sample Positioning System

SScanSS - Strain mapping, planning and visualization software

VULCAN - Materials Science and Engineering Diffractometer at SNS

Geomagic Studio 12 - a program which includes routines for capture of a large number of coordinate sets for the surface. This data is called a Point Cloud. Shape Studio also provide tools to take this point cloud and create a surface model which can be imported to CAD modeling software.

Datum - trusted feature such as a plane, circle, line, or point.

Fiducial Markers - Locations easily measured with the ScanArm or Laser Tracker for use in defining sample position 
Scan Arm - a coordinate measurement machine with both a point probe that measures $X Y Z$ points sequentially and a laser scanner that records thousands of $X Y Z$ points on the sample surface.

Laser Tracker - a metrology instrument that can accurately measure the XYZ coordinates of specific fiducial features on a sample using a SMR. 


\section{Chapter}

\section{"As-is" Model vs. CAD model for Sample Geometry}

SScanSS can support either an "as-is" model defined by the ScanArm or a CAD model that has been created in a CAD software package. In the case of a CAD model it is best to use the LaserTracker and CAM2 in the offline alignment laboratory to define the Sample Coordinate System (SCS) and to measure the fiducial points in the SCS.

\section{Choosing Model Type}

The CAD model is essential when measurement locations are associated with hidden or internal features which will not be captured by using the ScanArm to define surfaces. The CAD model can also be used along with the "as-is" model to determine distortions (using Faro's CAM2-X and/or Geo Magic's Qualify).

Choosing a model type dictates the instrument to be used. The Faro CAM2-X software supporting the laser tracker or hard probe on the ScanArm is more accurate and much less time consuming when using a CAD model. Therefore use of the LaserTracker and CAM2 software is the ideal methodology when using a CAD model. When using an "asis" model, GeoMagic and the Laser Probe on the ScanArm must be used. Chapter 7 is divided into two sections each detailing the different techniques; please refer to the relevant chapter for more information. 


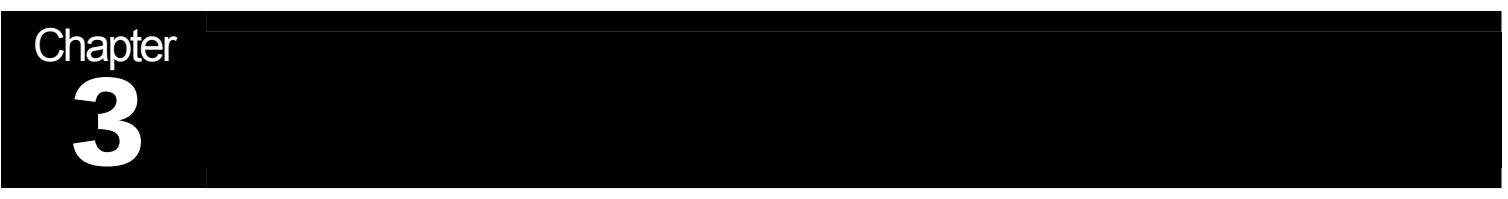

\section{Considerations for Establishing the Sample Coordinate System (SCS) and Defining Measurement Locations}

This section of the guide suggests ways to establish the sample coordinate system. While not essential, we recommend establishing such a coordinate system, as this is easiest for reviewing results and communicating subsequent measurements. The sample coordinate system is a very important part of the alignment process and must be considered carefully and established before any fiducial or point cloud measurements. The process of the definition of a SCS is very sample dependent. Be sure to take advantage of sample geometry and other features that can help facilitate easy measurements and relating the strain/stress information to the part geometry.

\section{Some Questions to Consider}

There are a few factors that need to be considered carefully when deciding the orientation and location of the SCS. Below are some guided questions that may aid in SCS definition.

- Does an engineering drawing of the part exist?

- If an engineering drawing exists then more than likely an origin has already been established and could be used for simple identification of measurement locations.

- Does sample geometry favor a specific coordinate system?

- Certain sample types can favor certain coordinate systems. For example the surface of a plate is well suited for defining the $X Y$ plane with $Z$ 
normal to the $\mathrm{XY}$ plane. A cylinder is also unique because a cylinder axis is readily defined.

- Do the measurement locations favor a specific sample coordinate system?

- A sample may have complicated geometry - such as a complicated curve on a gear tooth. If you want to measure the points normal to the tooth surface of this strange curve at specific depths some special coordinate system could be chosen to facilitate this action. However, the center axis and surface plane are likely more appropriate for defining the SCS of the gear. Note coordinates of measurement locations will be defined using SScanSS and working in a sensible coordinate system facilitates doing this.

- Datums are required to be measured for SCS definition. Therefore careful attention must be taken that all datums are easily measurable, are stable and firmly attached to mount or sample.

- Will the fiducial points be easily measured by the ScanArm and Laser Tracker when the sample is mounted on the goniometer?

\section{Marking Specimens}

It is important to make physical marking, with a marker, of the coordinate system on the part. This simple step can help avoid errors or confusion when trying to align the world coordinate system and the SCS. It is also very helpful to have a visual indicator as a permanent record on the sample as it will be moving from one location to another and often remounted on the goniometer multiple times.

\section{Considerations of Mapping Types}

In residual stress mapping there are three main types of mapping: 1-D, 2-D, \& 3-D. The different mapping types are applicable to different situations. A mapping type must be chosen that fits the particular sample type, sample preparation assumptions, and analytical needs.

\section{1-D Mapping}

Cylinders \& plates

\section{2-D Mapping}

Welds \& Compact Tension (CT) Specimens

\section{3-D Mapping}

Complicated Geometrical and Analytical Needs 


\section{Gauge Volume Considerations}

Gauge volume is an important consideration in defining measurement locations and mapping. The distance between measurement locations are in part dictated by the gauge volume size. It is important to remember that the strain measurement vector is along the diagonal of the gauge volume and not perpendicular to the sides. This means that the distance across the gauge volume is actually $a \sqrt{2}$ where "a" is the gauge volume width for $2 \theta=90^{\circ}$. The gauge volume within the horizontal plane is often viewed as a diamond with length of sides $=a$.

Mapping in the horizontal plane through the sample can be achieved by overlapping the edges of each "diamond" or by separating each edge from each other. It is recommended that the edges are overlapped for a better representation of the trends. By not allowing an overlap the data becomes coarser. The gauge volume in the vertical direction is rectangular. If measurement time permits, steps in the vertical direction should also overlap.

Sometimes it is also best to plan to collect measurement points needed to test for balance of forces. This could increase beam time overall but could better define the gradients of strain in the sample and serve as an excellent check that the experiment was performed correctly. Often this requires extending the number of point measured further from the region of interest.

\section{Creating a Measurement File}

Once a sample coordinate system has been established, a file containing the desired measurement locations of the coordinates may be created. The SScanSS simulation software is often the best choice for creating the file containing desired measurement location. An alternative is accomplished by creating a .txt file that is tab delimited in Microsoft Excel to organize the points. The points must be arranged so that each row of the Excel spreadsheet contains the $\mathrm{X}, \mathrm{Y}$, and $\mathrm{Z}$ coordinates of the measurement locations. The points need to be in the Sample Coordinate System (refer to chapter 7a for instructions). The example below illustrates how the points must be arranged.

Example: Points 1 - $\mathrm{N}$

\begin{tabular}{|c|c|c|c|}
\hline & $\mathrm{A}$ & $\mathrm{B}$ & $\mathrm{C}$ \\
\hline 1 & $\mathrm{X}_{\text {point 1 }}$ & $\mathrm{Y}_{\text {point 1 }}$ & $\mathrm{Z}_{\text {point 1 }}$ \\
\hline 2 & $\mathrm{X}_{\text {point 2 }}$ & $\mathrm{Y}_{\text {point 2 }}$ & $\mathrm{Z}_{\text {point 2 }}$ \\
\hline 3 & $\mathrm{X}_{\text {point 3 }}$ & $\mathrm{Y}_{\text {point 3 }}$ & $\mathrm{Z}_{\text {point 3 }}$ \\
\hline$\cdots$ & $\cdots$ & $\cdots$ & $\cdots$ \\
\hline $\mathrm{N}$ & $\mathrm{X}_{\text {point N }}$ & $\mathrm{Y}_{\text {point N }}$ & $\mathrm{Z}_{\text {point N }}$ \\
\hline
\end{tabular}


After the Excel file is created, it must be saved with a ".measurement" file extension. Simply select a txt file type when saving the file, then change the file extension to ".measurement" by typing ".measurement" after the filename.

Example: filename.measurement 


\section{Chapter

\section{Use of 80/20 in Sample Mounting}

The 80/20 hardware allows for easy mounting the sample at the alignment laboratory, NRSF2, and VULCAN due to common aspects of each. As expected, sample mounting depends heavily on the specific sample. This chapter merely provides some tips for sample mounting and for avoiding issues that could adversely affect an experiment.

\section{What is $80 / 20 ?$}

The $80 / 20^{7}$ system is a collection of rectangular rods of aluminum of differing length with holes set at $25 \mathrm{~mm}$. The rectangular pieces also have T-slot tracks with T-slot nuts that can move freely until a standard M6 bolt is attached from one piece to another. This attachment mechanism is very strong and maintains orthogonality between the mounted pieces with ease. Other 80/20 pieces like angles and larger cross section pieces are also used. The system is similar to a child's Erector or Lego play set and is infinitely adaptable.

\section{Common Aspects of Mounting System}

The real strength in the mounting system is the interchangeability of the $80 / 20$ mount between the indexer table in the alignment lab, NRSF2 and VULCAN. The table top as well as each of the instrument Sample Positioning System's (SPS) tops have a hole pattern of M6 holes set on a $50 \mathrm{~mm} \times 50 \mathrm{~mm}$ grid. This mounting scheme will allow the sample to be mounted in the alignment lab, aligned, and then carried to the instrument and mounted directly onto the SPS.

\section{Mounting Considerations}

Certain sample types favor certain mounting schemes. This is important to consider when building a sample mount. For example, in a plate it is best to keep the $80 / 20$ pieces in the plane of the plate. This will keep mounting hardware away from the slits and reduce risk of collisions. If possible have several M6 holes drilled and tapped along the edges of the plate. Holes should be at multiples of $25 \mathrm{~mm}$ and parallel with the edges of the plate. 
Also vibrations must be considered. A mount consisting of one piece of $80 / 20$ would be less of a collision risk but could allow for vibration of the part. It is important to make the mount as stiff as needed to retain a rigid mount and to avoid possible vibration issues.

\section{Mounting Examples}

Below are some pictures (Figures 1\&2) of some example mountings that are possible from using the $80 / 20$ system.

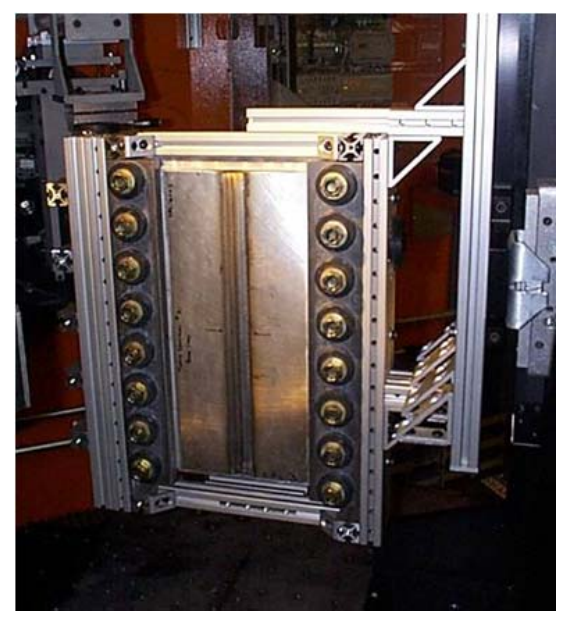

Figure 1. A Plate is mounted within a frame built with $80 / 20$ - notice the hardware is in the plane of the plate. The frame readily is attached to the SPS.

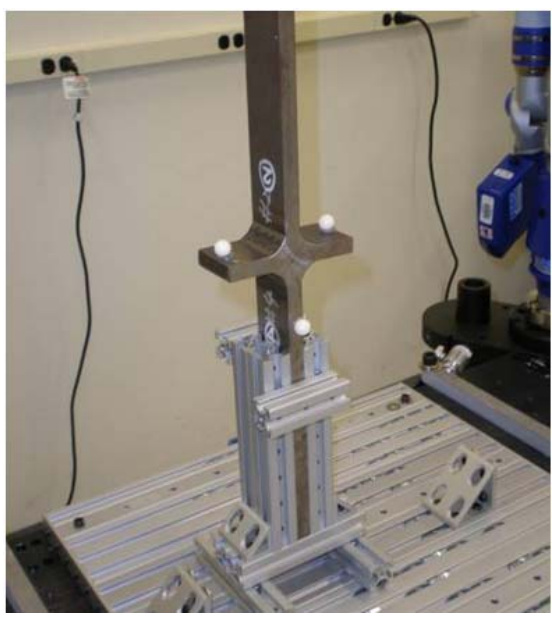

Figure 2. The $80 / 20$ hardware is used to mount an oddly shaped part for easier measurement.

\section{D-Zero Reference Coupons}

The strain free interplanar spacing coupons, also known as d-zero reference coupons also need to be attached to the sample mount. Some consideration should be given as to where these small samples will be attached. For example, you desire the $d_{0}$ sample to be near the "stressed region" of interest, so that excessive SPS stage movement is not required (collision risk). However, not too close to the stressed region of interest, such that the diffracted signal from either is attenuated by the other. Often hot glue, super glue or red wax can be used to achieve a secure attachment and a favorable location while avoiding adding extra hardware to mount the $d_{0}$ coupon. One consideration with the hot glue, red wax, or other hydrocarbon materials, is to avoid placing the hot glue such that it would interfere with the neutron beam path, as the hydrogenated nature of these glues causes increased background signals and attenuates the neutron beam. 


\section{Chapter}

\section{Mounting Sample on Indexer}

Table and Adding Fiducial Points

The sample must now be mounted on the indexer table in preparation for ScanArm or LaserTracker measurements. The fiducial points are used as reference points that can quickly be measured and used to define the sample orientation at any mounting on an instrument. Fiducial points are an important part of alignment and careful forethought must be observed when choosing what type or types of fiducials to use. This chapter presents advantages and disadvantages of each point type.

\section{Sample Mounting Considerations for Scan Arm Measurement}

- Mount the sample on the offline indexer table in such a way that the ScanArm can easily "see" and "reach" each side of the sample and mounting hardware.

- The SCS's X, Y, and Z axes do not have to be in any particular orientation. The coordinate system can be defined by the LaserTracker later so there is no particular reason why the sample has to be orthogonal to the table for example.

\section{Fiducial Points}

At least three non-collinear fiducial points are required for alignment. We recommend four or more points be used for additional accuracy and also for flexibility in case one or more is not observable or has to be removed.

\section{Choosing Fiducial Types}

There are four separate methods of defining fiducial points. Each method or type has advantages and disadvantages. 


\section{Magnetic Nest and Spherically Mounted Retroreflector (SMR)}

SMR nests are mounted onto the sample or $80 / 20$ mounting frame using hot glue. With the ScanArm, a sphere or an SMR is placed into the nest and points on the outside of the sphere are measured. SMR nests contain magnets which hold the retro reflector firmly in place. If the ScanArm hard probe is used, be sure not to dislodge the SMR from the nest. From multiple points about the SMR, GeoMagic Studio 12 software is used to fit a sphere mode and thus to define the center of the SMR. With the Laser Tracker, the SMR center is directly measured using CAM2 software. This is the best method and most commonly used. Unfortunately a LT-CAM2 measure can only be input to Geo Magic Studio by use of the keyboard..

\section{Advantages}

- Reproducible with ScanArm and LaserTracker

- Useful to put both the ScanArm and LaserTracker into the SCS

- Nest are easily attached and removed without damaging part or adding macro stresses

- Human error is minimal

- Measurement of point locations is fast, reproducible and accurate.

\section{Disadvantages}

- A clear line of sight must exist between the SMR mounted in the nest and the laser tracker

- Nests could be a collision risk, require greater slit offsets, or shadow the neutron beam

\section{Sample Geometry}

Sample geometry uses features of the part that can be turned into points, like three intersecting planes to define a fiducial point, the corner. For a cylinder the intersection of the cylinder axis and the plane for the end define a point. This method is most often used to define points associated with the SCS origin.

\section{Advantages}

- Does not require any additional hardware to be attached

- Helpful for small parts

\section{Disadvantages}

- Subject to human error

- Sample deformations (e.g. non planar surface assumed to be planar). 
- Requires more measurement time and subsequent construction of the points using CAM2 or GeoMagic software.

- Mounting hardware may obscure the positions of the sample used to define a plane or axis.

\section{Center Punch}

Center punching changes the sample by impacting the surface to leave a small indent that can be used to define a point. This point is defined using an extension rod with point on one end and SMR nest on the other to define a sphere using the laser tracker. The center of that sphere becomes the fiducial point. This method should be used as a last resort as we have observed larger errors in defining the point compared to methods 1 and 2. Operator training and practice have proved to be essential, but errors still can be made due to the point end slipping/walking on the surface.

\section{Advantages}

- Does not require any additional hardware to be attached

- Keeps hardware away from beam and slits

\section{Disadvantages}

- Indentation damage on sample surface may not be acceptable

- Measuring with ScanArm is difficult and requires experience and care

- Not as reproducible as other measurements

\section{Fiducial Balls}

Fiducial balls are precise spheres that can be attached to the sample or the $80 / 20$ mounting using hot glue (see Figure 4). From a point cloud data set a sphere is constructed and the center is the fiducial point. Alternately, the hard probe of the ScanArm can be used to define a number of points about the sphere. Use of fiducial balls is a sound method that is commonly used.

\section{Advantages}

- Reproducible accurate measurements of sphere center point with ScanArm point probe and LaserTracker

- Easily attached and removed without damaging part or adding macro stresses

\section{Disadvantages}

- Fiducial balls could be a collision risk or limit slit offsets or shadow the neutron beams 


\section{Chapter}

\section{Equipment Start-Up \& Calibration}

The following is a step-by-step guide to the use of the FARO Laser Tracker and ScanArm with ball probe. A flow chart of all the recommended steps is provided in Appendix $A$.

1. The ScanArm with GeoMagic Studio $\mathbf{1 2}$ is to be used to:

i. Define a SCS and

ii. Create the surface model or read in a CAD 3-D model

2. The Laser Tracker with CAM2 cannot create a 3-D model but is occasionally used for metrology and training for its use at NRSF2 and Vulcan.

\section{Laser Tracker Equipment Start - Up}

Be sure that the LaserTracker is connected to the power supply. The power supply is the small blue box under the computer table connected to alignment laptop. Open CAM2 software on the laptop. Power on the Laser Tracker by flipping the switch located on the power supply. The tracker will go through a warm-up procedure. The details of the warmup will be displayed on the computer screen.

\section{ScanArm Equipment Start - Up}

Power on the ScanArm by flipping a switch on the base of the arm assembly. Start the alignment laptop on the desk, start GeoMagic Studio 12 by clicking the shortcut on the desktop. The Faro ScanArm, in a laboratory setting, will need about 15 minutes to warm up for stabilization. The ScanArm initializes each encoder by the user physically rotating each joint (Figure 6).

\section{Laser Tracker Equipment Calibration}

The Faro Laser Tracker will warm up and self-calibrate after launching the measurement software CAM2. The CAM2 World coordinate system origin is within the Laser Tracker with $\mathrm{XY}$ horizontal and $\mathrm{Z}$ vertical as defined by gravity. 


\section{ScanArm Equipment Calibration}

The ScanArm will need to be calibrated if the arm has been offline. In Geomagic Studio 12, go to > Hardware Tab>FARO PLUGIN 15.0. A dialog box will appear on the left side of the screen (Figure 3). Click the ball probe icon (see Figure 3) and click the calibration icon (the picture of the probe with the "?" beside it). In this menu we can calibrate both the ball probe and the laser probe (Figure 4A).

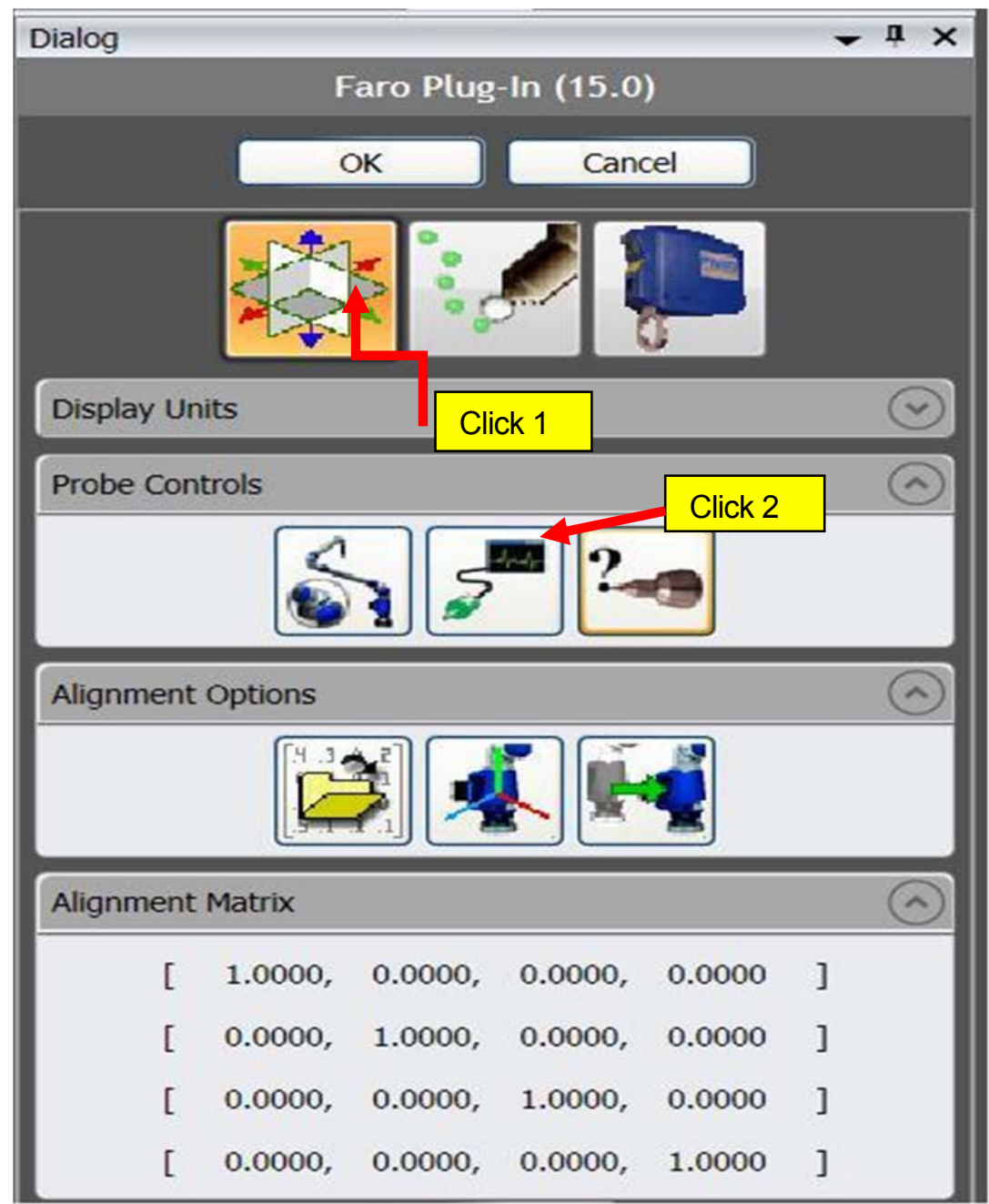

Figure 3. Hardware Dialog Menu 


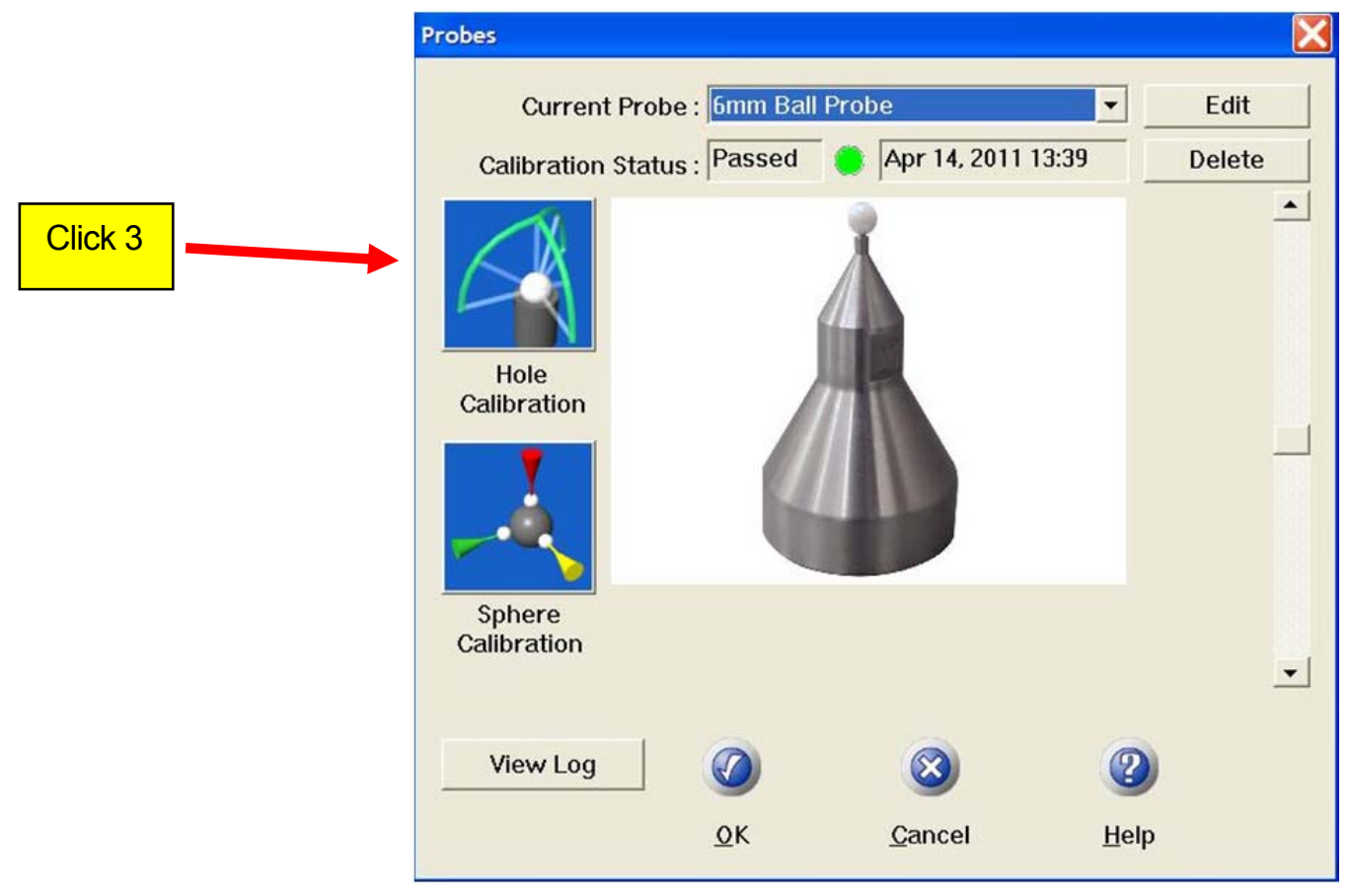

Figure 4A. Probe Calibration Window

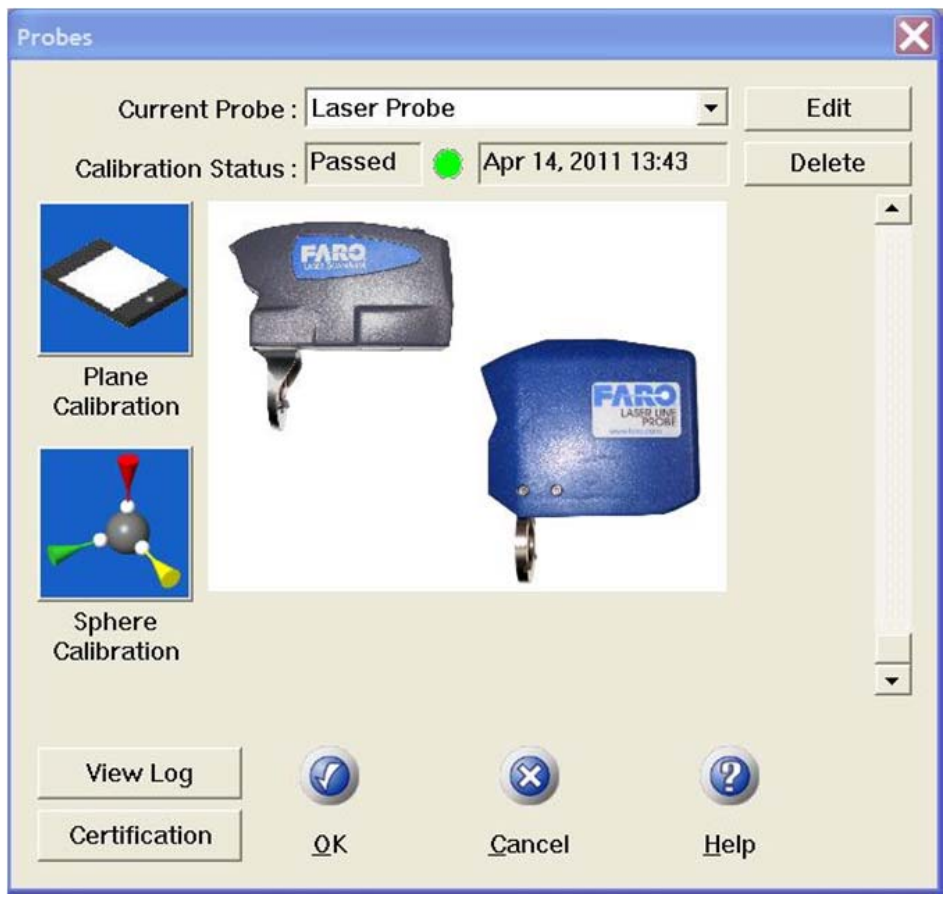

Figure 4B. Laser Probe Calibration Window 


\section{Ball Probe Calibration}

In the calibration window (Figure 4A), Current Probe Box, select the "6mm Ball Probe" and then select the "Hole Calibration" option on the left side of the dialog box (arrow). Follow the onscreen instructions to calibrate the scan arm's ball probe using the probe calibration fixture on the indexer table. Click OK when finished.

Note that if the arm has been offline then you must move the arms to all extents of movement to initialize the encoders of the joints of the arm. If this needs to be done a screen will appear with a picture of the scan arm and arrows denoting the motion of each joint of the arm (Figure 5). Just move the arm until all red arrows on the displayed diagram disappear.

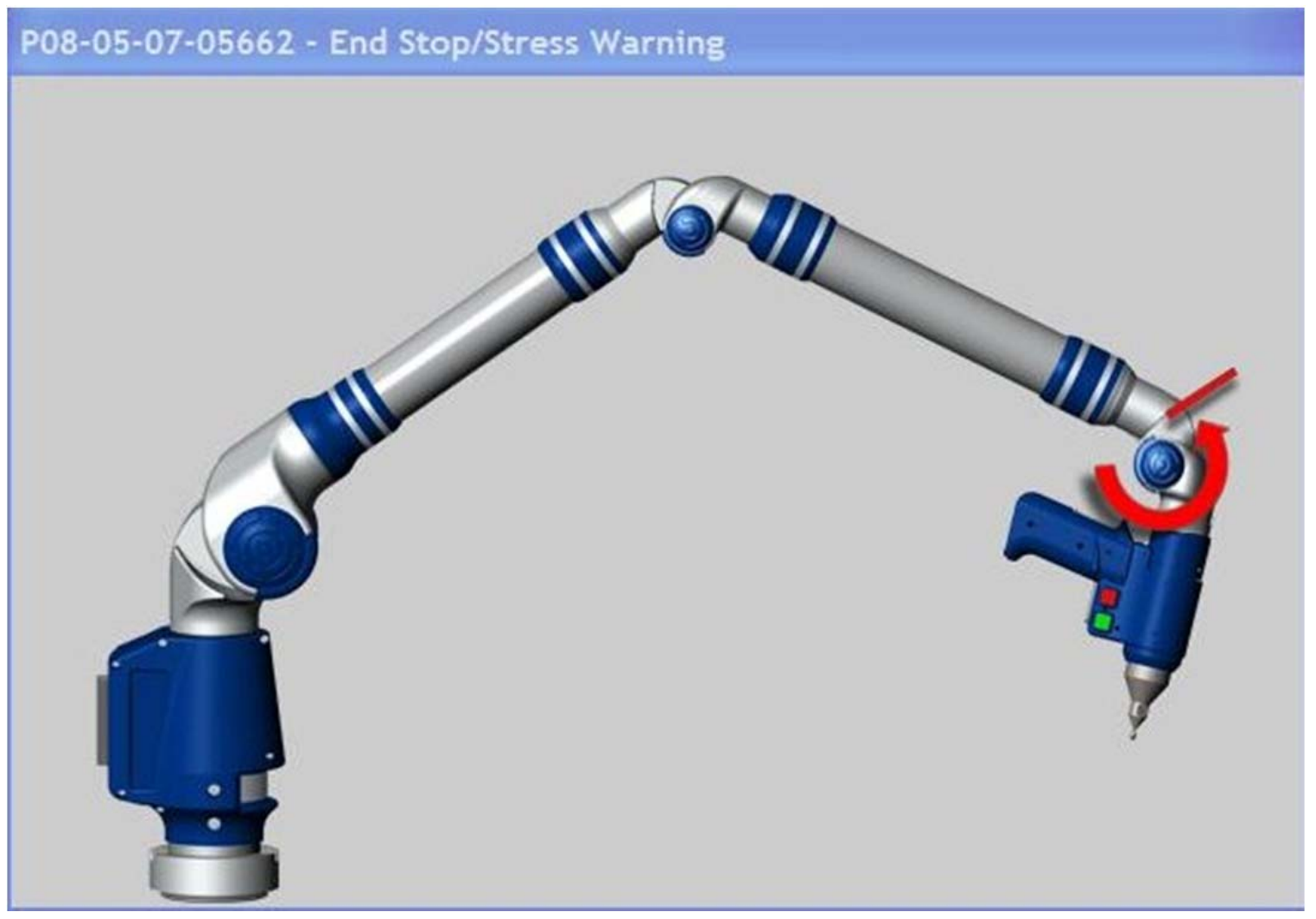

Figure 5. Exercising ScanArm Joints 


\section{Laser Safety}

Both the Laser Tracker and ScanArm laser probe use a low power laser as part of the measurement system. While these are rated Class 2 (low-energy, not harmful), do not look into the beam.

\section{Laser Probe Calibration}

Now that the ball probe is calibrated, you must calibrate the laser probe. Simply select the scanner as an option in the "Current Probe" pull down menu (Figure 4B). Select the plane calibration option on the left side of the box. Then select $>$ measure plane with ball probe $>$ click YES to "auto exposure" > click OK to "Measure plane with current ball probe" > follow the onscreen instructions to calibrate the laser probe (it is best to use the black and white calibration block for this step). During calibration you must capture the point with tapping the green button on the arm and tapping the red button to move to the next step. Click OK when finished.

Scan density must be set to a 1:1 ratio for proper calibration (this is default setting).

\section{World Coordinate System}

When the arm has completed calibration it will be in the ScanArm's world coordinate system with the origin at its base. This coordinate system should be changed to the sample coordinate system by following the steps described in chapters 3 and in chapters7a or $7 \mathrm{~b}$. 


\section{$7 \mathbf{a}$}

\section{Transforming From World to the Sample Coordinate System (SCS) with ScanArm/GeoMagic}

There are multiple ways to define coordinate systems in Geomagic. In this guide you will learn a flexible method that can be used in almost all cases that involve defining datums and then "pairing" those datums with coordinate features. This chapter is a quick overview followed by a detailed description of the important steps.

\section{Overall Procedure}

1. In Geomagic after choosing "new" project - Click Hardware tab > FARO plug-in tab

2. Define as many datums (planes, lines, points) as needed to define a coordinate system that is convenient (Figure 6). A datum can be measured directly or constructed from measured datums. Click > Ball Probe Icon > Plane icon (row 2, column 3 of Figure 7) $>$ With ScanArm capture points on desired plane, minimum of 3 points, press the green button on the Scan Arm to capture a point and the red button when the desired number of points on the plane are completed. 


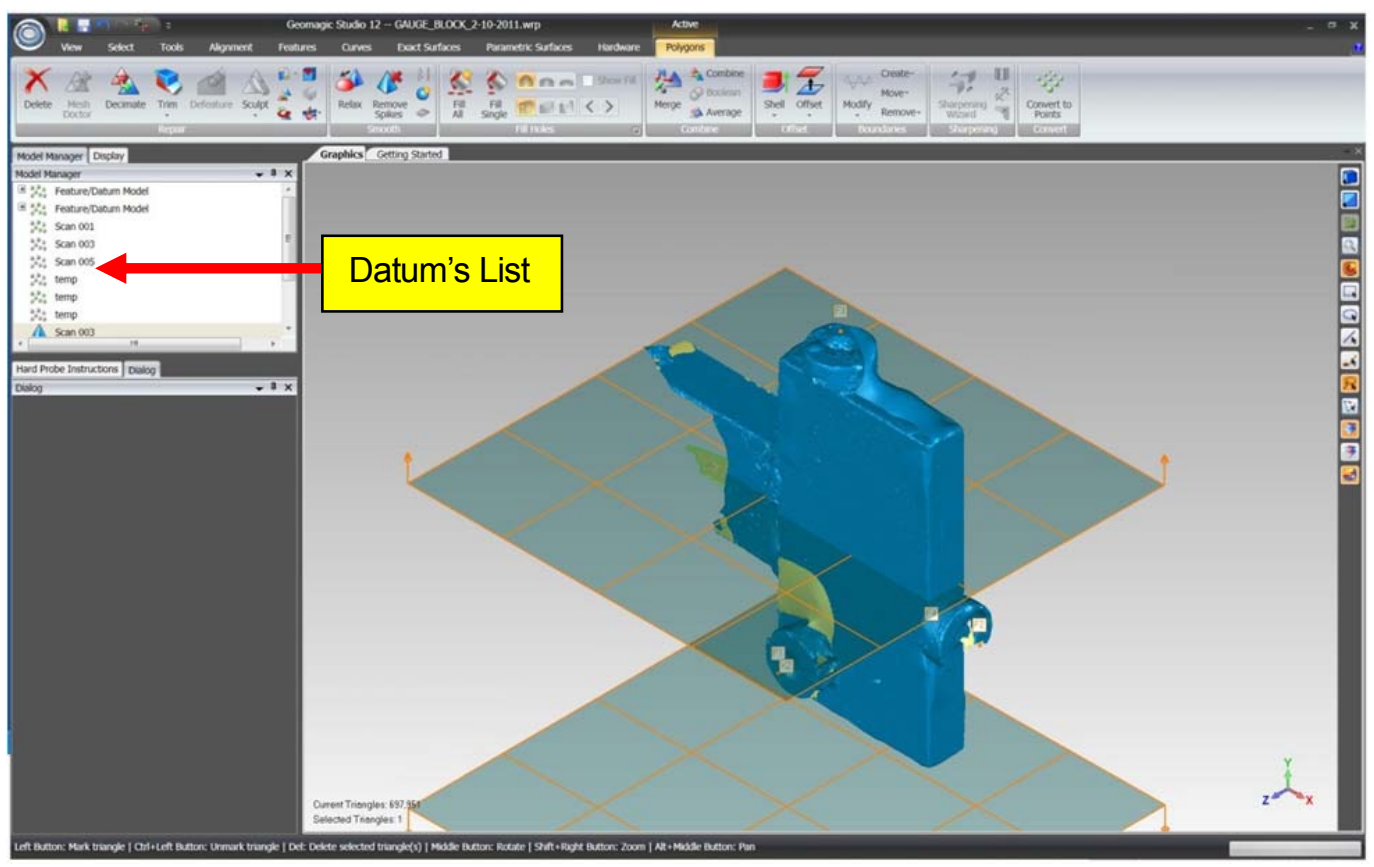

Figure 6. GeoMagic Modeling Window

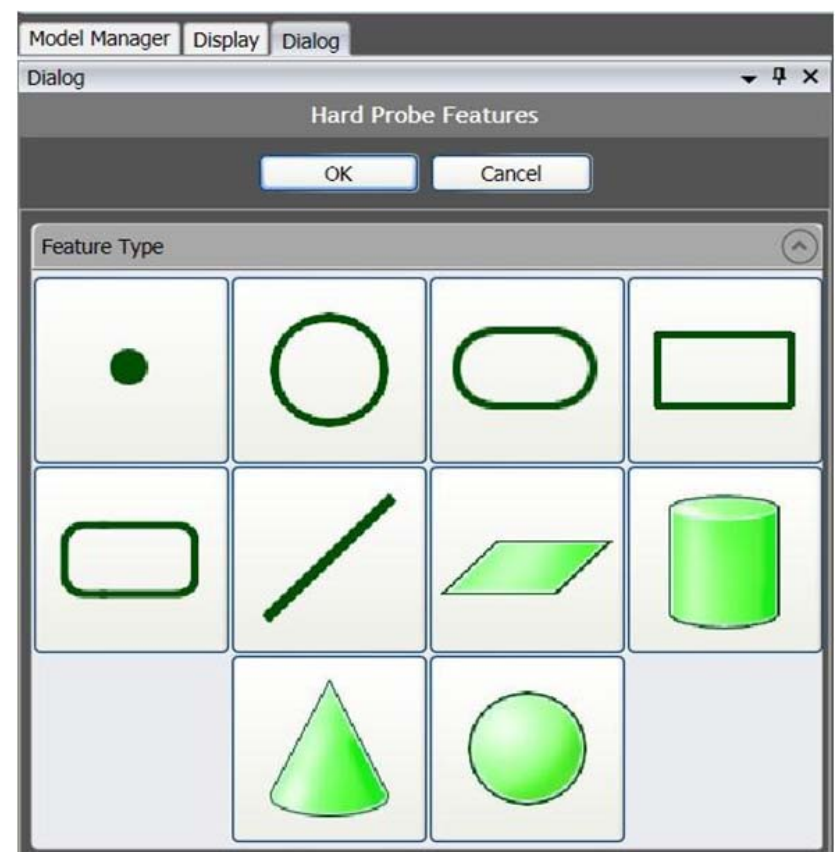

Figure 7. Plane Measurement Icon

3. Use the Alignment tab > Align to World option to align the world coordinate system to your desired SCS. Alignment is achieved by pairing the measured datums to known world features such as planes, coordinate axis, or the origin (Figure 8). The first pair will take priority. 


\begin{tabular}{|l|l|l|l|}
\hline Model Manager & Display & Dialog & \\
\hline \hline Dialog & & \\
\hline
\end{tabular}

Figure 8. Alignment Window for Pairing

\section{Defining Datums}

\section{Datum Types}

The main types of datums that will be used are planes, circles, lines or axes, or points. Each of these are defined by the ScanArm ball probe and are selected from the Hardware tab>FARO PLUG-IN tab. Keep in mind that you must have enough datums to have a fully constrained coordinate system. Frequently used is a plane, a line and a point (referred to as 3-2-1).

An example of a datum to be used in alignment would be using the top plane of a plate. One can measure the top plane of the plate and then define it as the XY plane. 


\section{Measuring Datums}

To measure a datum, go to Hardware tab>FARO PLUG-IN tab and select the ball probe option (second from the left, see Figure 1). From this menu you can define a set of datums that can be used to align the world coordinate system of the ScanArm to the coordinate system you wish for the sample.

\section{Constructing Datums}

Datums can also be constructed from measured datum points and features. This means a point can be derived from an axis intersecting a plane, two intersecting planes can define a line (axis), etc.

A good example of a constructed datum would be first, measuring a point you wish to be the origin and then measuring a plane that should be perpendicular to a certain axis. Then by choosing Features tab you can select the axis icon then click construct from a point and a plane icon. Then you have a new axis that intersects the origin and is perpendicular to the plane you measured. This can be very helpful for sample coordinate system definition. 


\section{Aligning ScanArm's World Coordinate System to SCS}

To use the defined datums to align the coordinate systems simply choose Alignment tab> Align to World tab (Figure 9A). In this dialog window it will give you a list of the defined datums you have measured as well as coordinate system features ( $X$ axis, $X Y$ plane, origin, etc.).

To align the system simply select the datum you wish to be a certain coordinate system feature and click the pair button (Figure 9B). For example; if you measured PLANE_01 as the $X Y$ plane on the sample you would pair it to the $X Y$ coordinate plane in the program. This will constrain the two together. The dialog box will tell you when the coordinate system is fully constrained then simply click accept and you are ready to move on to the next step.

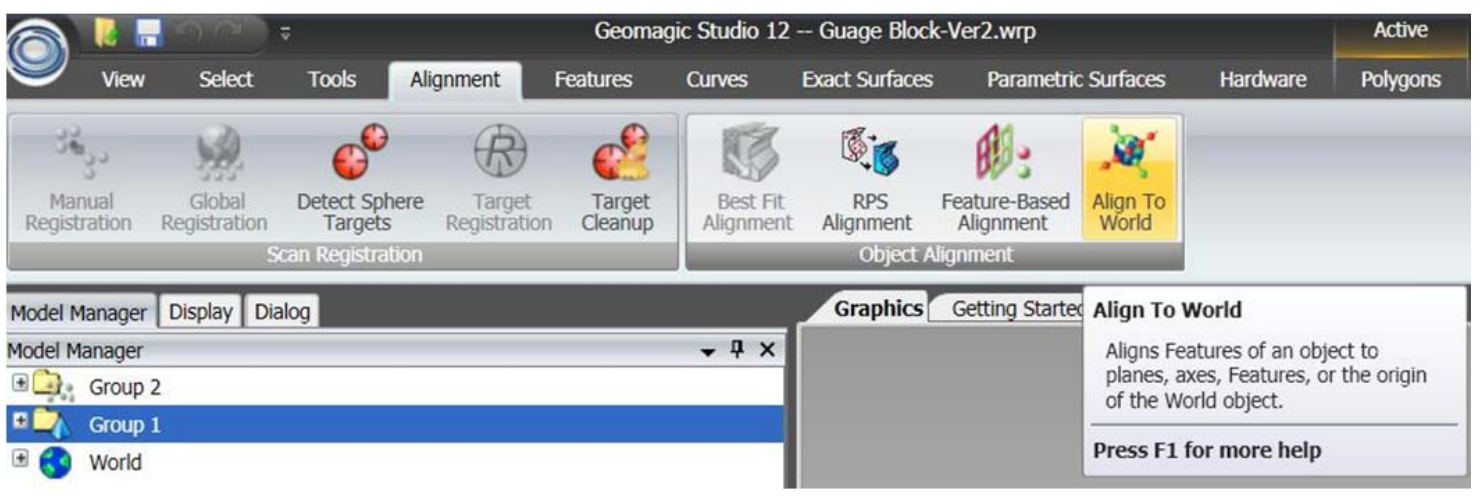

Figure 9A. 3-2-1 Construct Coordinate 


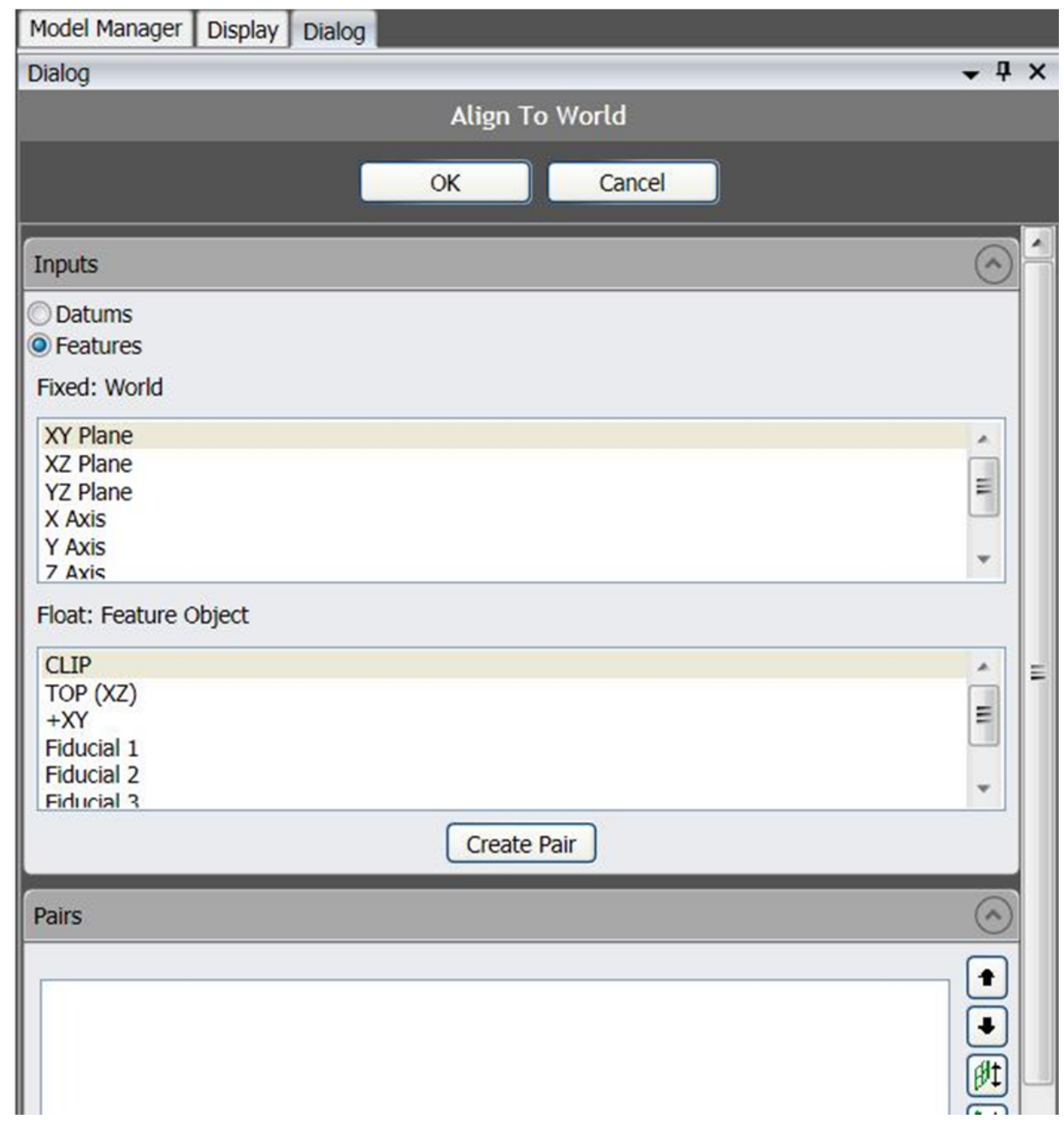

Figure 9B. Plane Alignment Dialog

Note that the top pair will take precedent over the lower pairs. This means that it will be the primary alignment. It is best to always define a plane as the primary. With a plane being primary, GeoMagic will then project any axes or points that are off the plane onto the plane. 


\section{Chapter \\ $7 b$}

\section{Transforming From World to the Sample Coordinate System (SCS) with FaroLaser Tracker and lor ScanArm ICAM2-X Software}

The thought process behind alignment in CAM2 is the same as in GeoMagic. The only difference between the two processes is the user interface provided to align the datums to the coordinate system features. This section is merely going to show the procedure of how to measure and then align to the SCS. Similar considerations of datum definition are listed in Chapter $7 a$.

\section{Defining Datums}

Datums in CAM2 function the same as in GeoMagic. To measure datums simply click the Measure button and one of the many different options for measurement (Figure 10). Follow the onscreen instructions for the individual measurement option. For additional questions refer to the CAM2 manual or help file. 


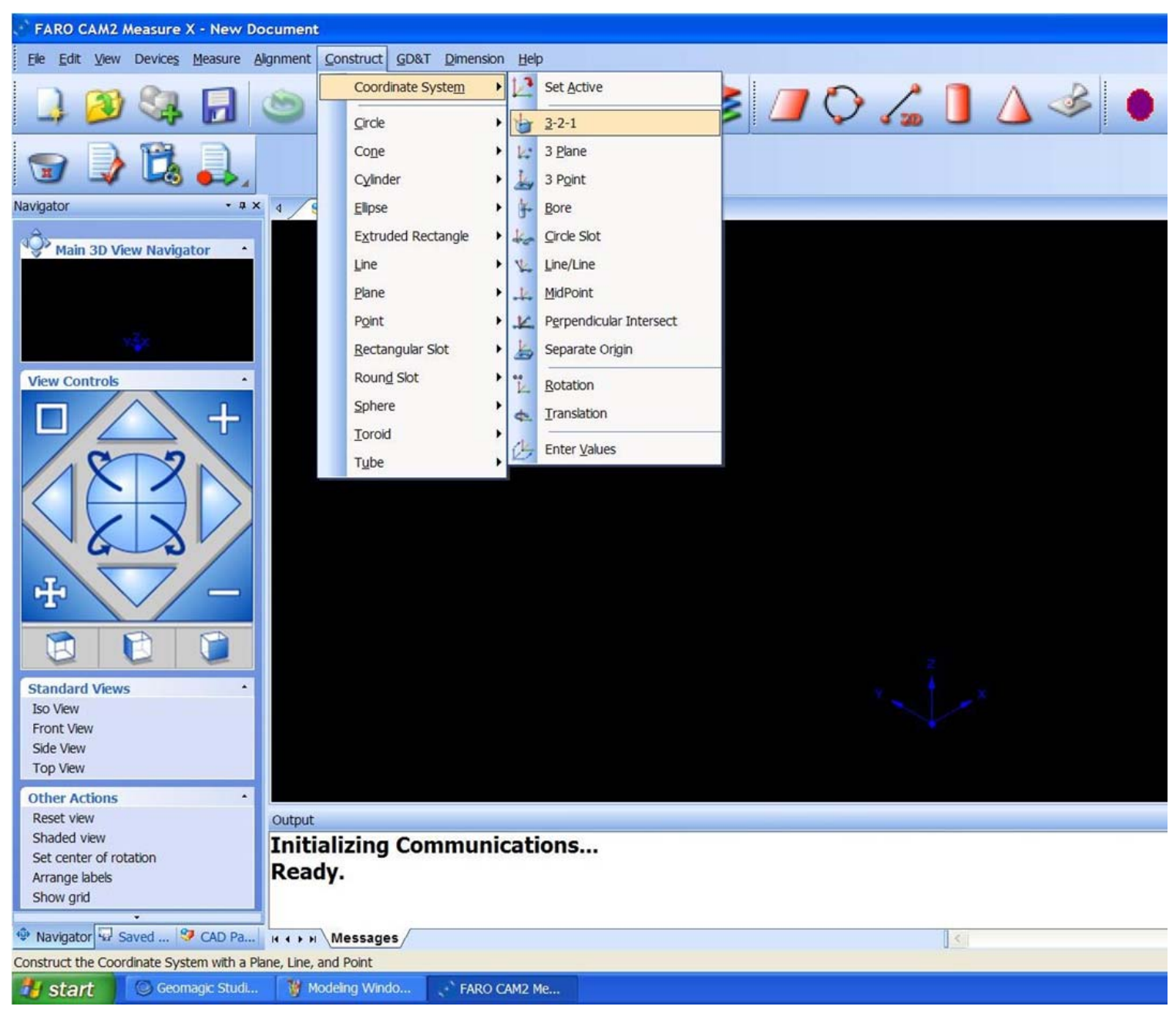

Figure 10. CAM2 Options Window

\section{Aligning LaserTracker to SCS}

\section{SCS Construction}

Alignment is achieved similar to the techniques used by GeoMagic.

Click "Construct "> "Coordinate Systems" from the top menu and choose the coordinate system you wish to use (Figure 10). This could be a $3=$ plane, $2=$ line, and $1=$ point.

In the dialog box that appears use the pull down menus to select the datums you wish to define as coordinate features and then click OK. 
Alignment/CAD=Part

This aligns the FARO world coordinate system to the chosen sample coordinate system. The XYZ values measured with the FARO device must be in the sample coordinate system as you have defined for the samples. This method is rarely used.

\section{Constructing Datums}

As in GeoMagic, datums can be constructed from measured datums. This means you can construct a line (axis) from two measured points or as the perpendicular line from a point to a plane.

Simply click "Construct" and select from the options presented. Each menu is different depending on the construction selected so follow the onscreen instructions for each. 


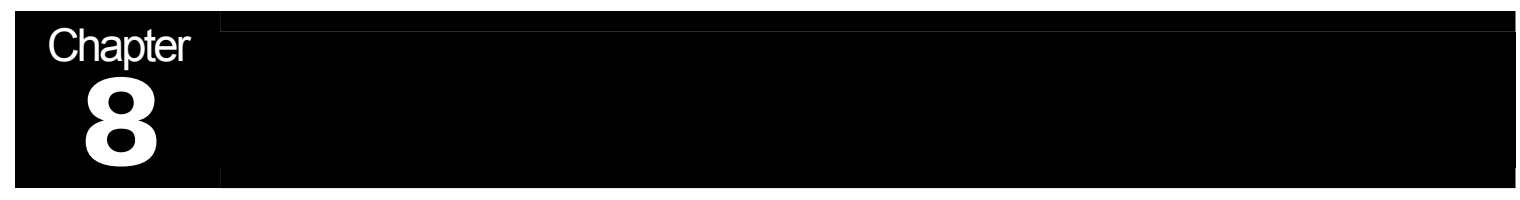

\section{Measuring the Fiducial Points in the SCS}

The fiducial points will be measured twice. They are measured initially in the SCS and then again in the instrument coordinate system. The measuring technique will vary depending upon the fiducial type, but most often one uses SMR spheres to define the center of the sphere as a point. This chapter is for measurement of fiducial points in the SCS with the Faro Scan Arm.

\section{Measurement Procedure}

Put SMR spheres into the fiducial point nest. Alternatively, fiducial spheres could be attached to the sample and /or mounting frame.

In Geomagic select the Hardware tab, then select the Ball Probe Features button, the Feature Type window should appear (Figure 11). In the Feature Type window select the sphere measurement button. Use the procedure listed on the screen and defined in the scan arm manual. 


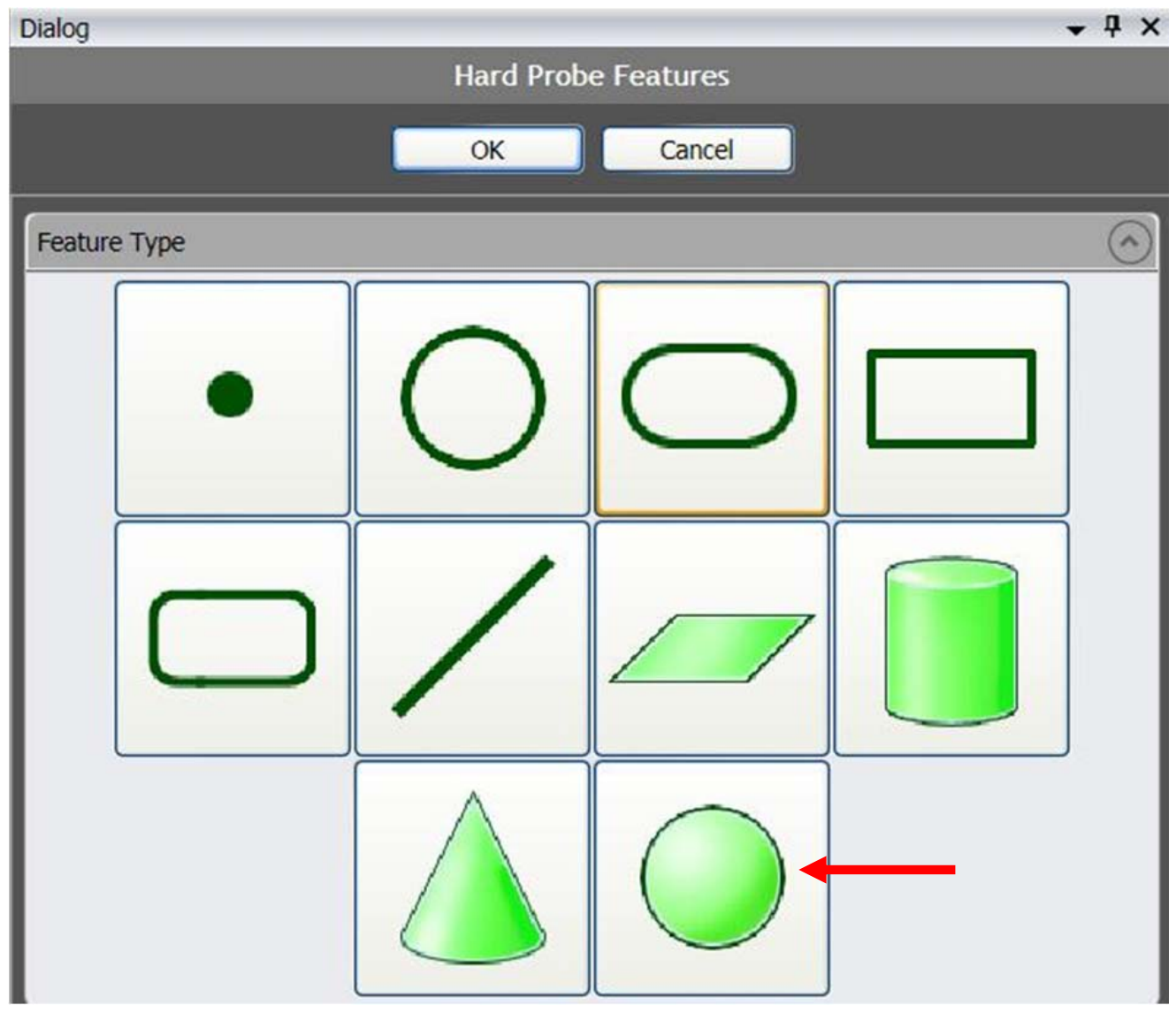

Figure 11. Feature Type Window

At least 4 points are needed to define a sphere. These are obtained by touching the touch probe to the surface of the SMR/fiducial ball. It is recommended that $6-10$ points be measured to achieve good reproducibility in the measurement of the center of the sphere. Once all SMR measurements have been completed select the Model Manager window to ensure that all spheres were measured.

Now select the Features tab, then the Point feature; in the dropdown box select Parameters (Figure 12), the Create Point dialog box should appear (Figure 13). 


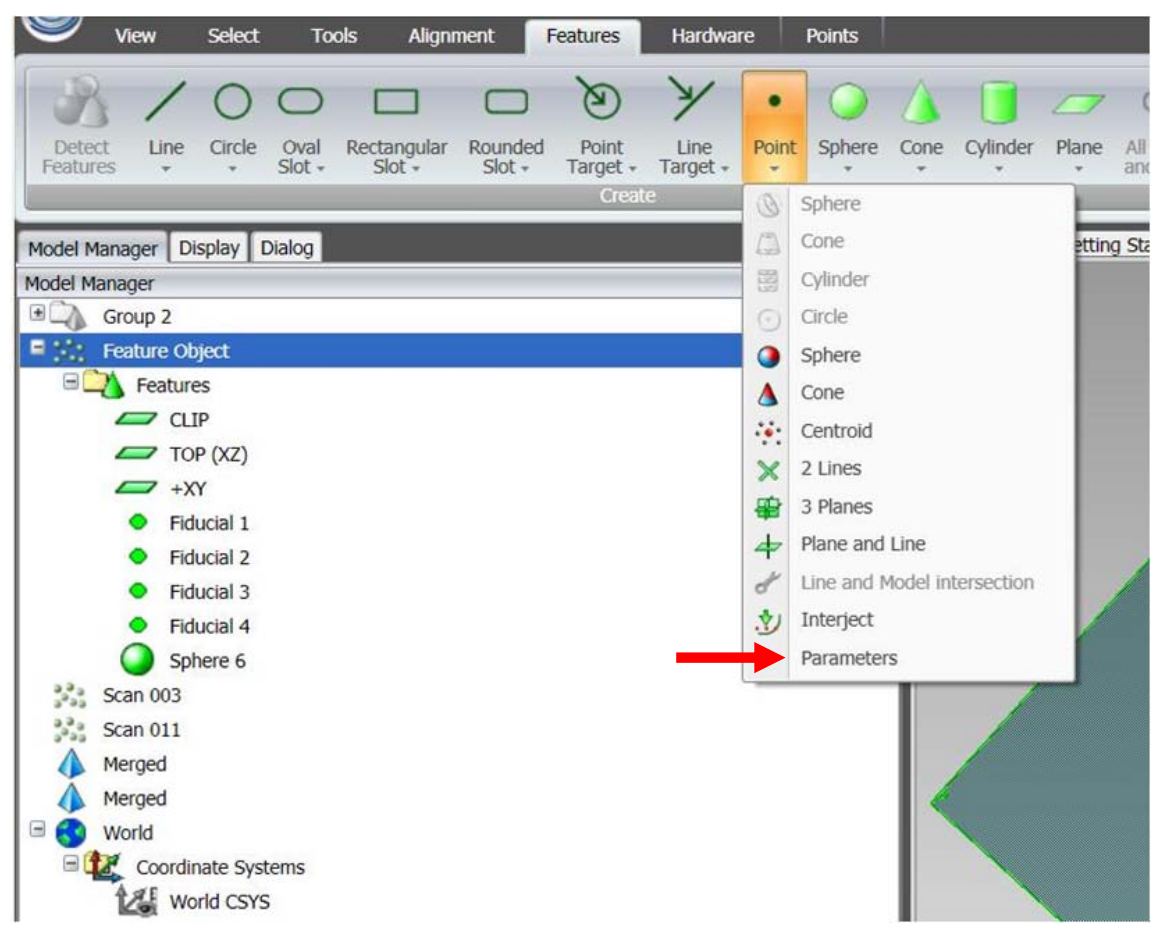

Figure 12. Fiducial Path

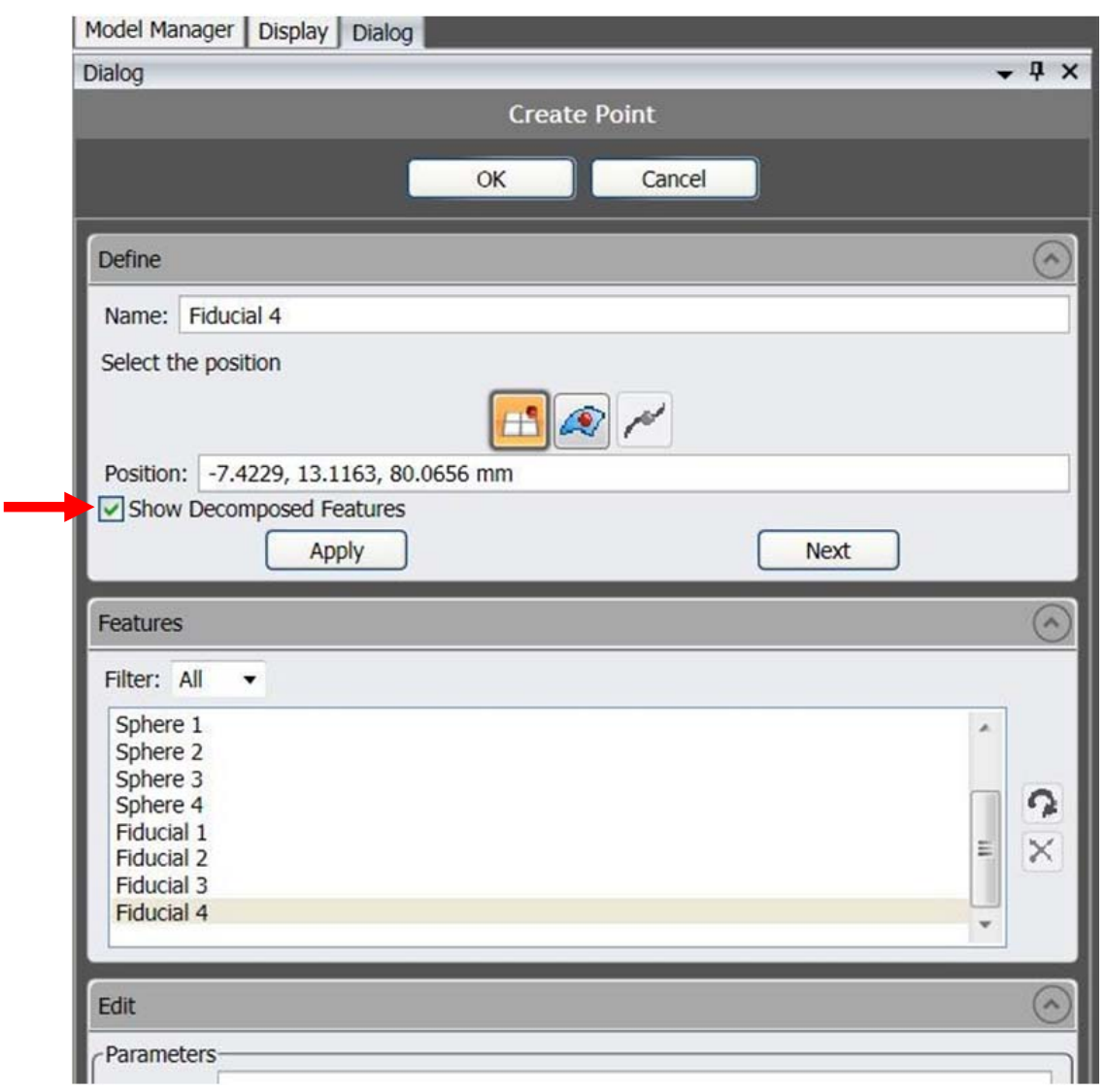

Figure 13. Create Point Dialog 
In the Create Point dialog box select the Show Decomposed Features check box. In the Graphics display various measured features are shown. For spheres the naming convention is "Sphere \#-Center". In this window, select the desired sphere using the mouse.

In the Dialog window name the point appropriately, click apply then next.

Note, the point/center position information is listed in the Dialog window and may be recorded. Repeat for all spheres, and then click OK when finished. Now the spheres may be deleted from the Feature Object pane in the Model Manager window.

In addition to the method listed above, point coordinates may be viewed by right clicking on the object i.e. "Fiducial 1" and selecting properties(Figure 14).

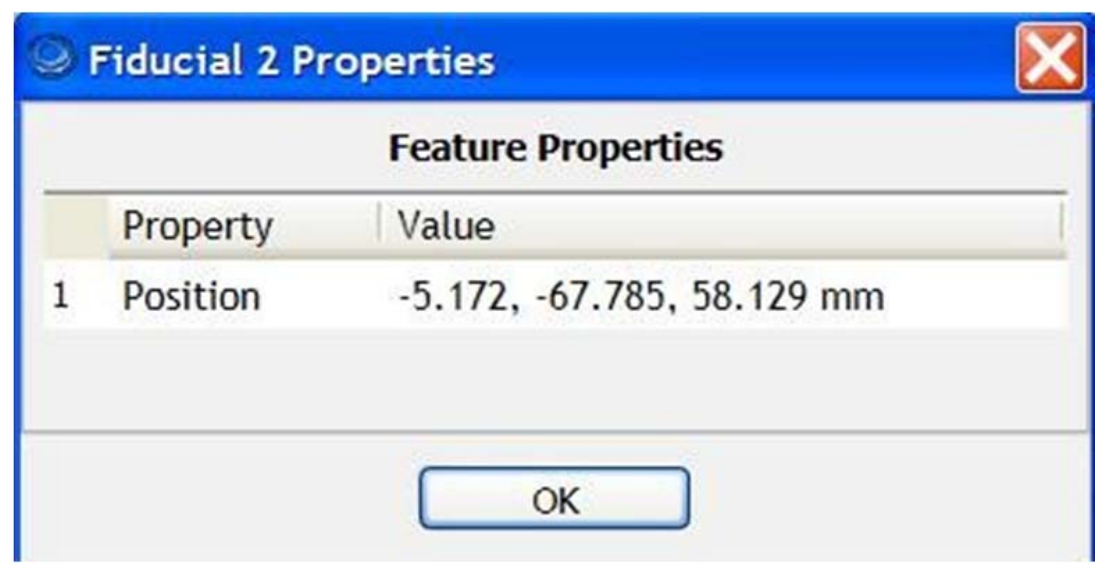

Figure 14. Fiducial XYZ

Example for Recording Fiducial Points:

\begin{tabular}{|r|r|r|r|}
\hline 1 & $\mathrm{X} 1$ & $\mathrm{Y} 1$ & $\mathrm{Z} 1$ \\
\hline 2 & $\mathrm{X} 2$ & $\mathrm{Y} 2$ & $\mathrm{Z} 2$ \\
\hline 3 & $\mathrm{X} 3$ & $\mathrm{Y} 3$ & $\mathrm{Z} 3$ \\
\hline
\end{tabular}

After the Excel file is created, it must be saved as a ".fiducial" file extension. Simply select a .txt file type when saving the file, then change the file extension to ".fiducial" by typing ".fiducial" after the filename.

For example - "filename.fiducial" 


\section{Chapter \\ 9}

\section{Obtaining "As-Is" Surface Model for the Sample}

This section details how to construct an "as-is" CAD model. This model will take into account deformations and give an accurate representation of the mounting hardware as well as the sample. The ScanArm obtains $X, Y$, and $Z$ valves for a point on the surface of the part and mounting hardware using a laser scan probe. This large collection of points is called a "point cloud." If a CAD model is to be used then there is no need to use the ScanArm laser probe to produce an "as is" model.

\section{Scanning Procedure}

Before scanning, be sure the ScanArm is calibrated. Follow the procedure detailed in Chapter 6.

In the FARO Plug-in dialog box, click the laser probe icon (Figure 15). 


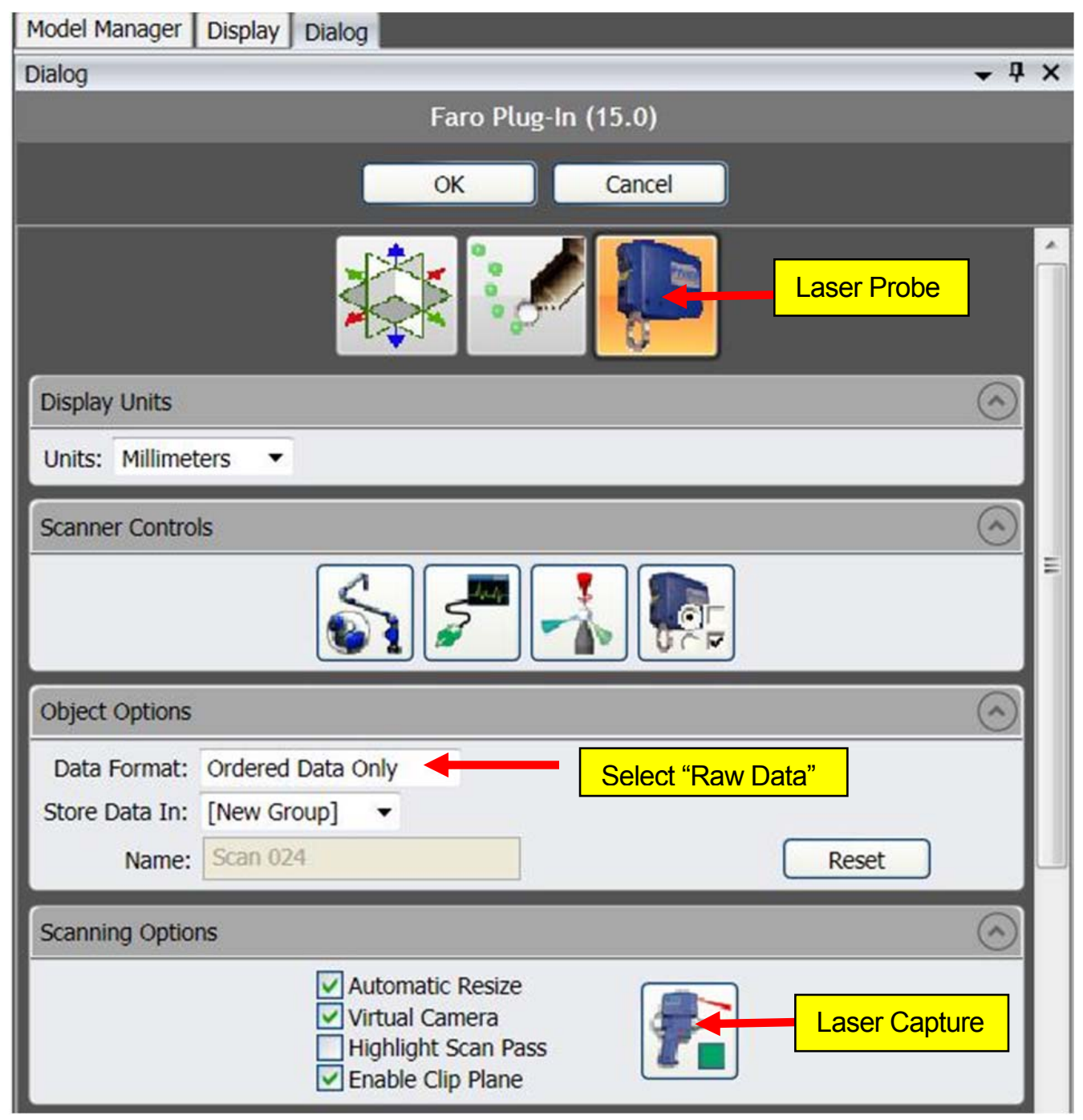

Figure 15. Laser Probe Dialog

Under Objects Options select "Raw Data Only" in Data Format drop down menu (Figure 15).

In the Laser Probe dialog box under Scanning Options click Automatic Resize, Virtual Camera, and Enable Clip Plane (Figure 15).

In the Advanced Options section (Figure 16) select Remove Outliers, Remove Overlap, Reduce Noise, and adjust noise slider bar to desired level (medium-high).

Note: Before any scanning, you should power on the indexer table via the power box to prevent the table from unnecessary rotation. If the indexer table is powered off do not lean on the table because it may move. 


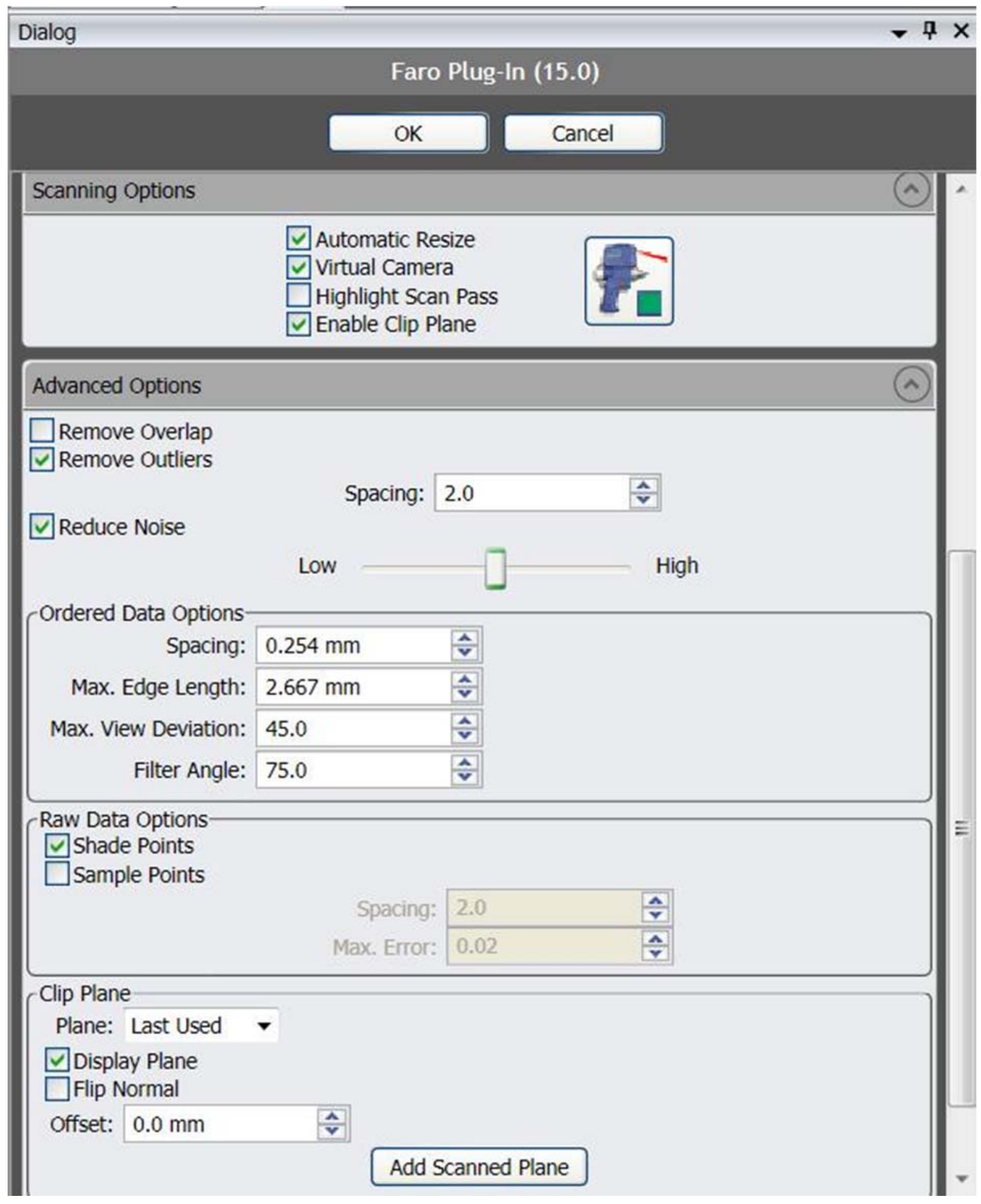

Figure 16. Laser Probe Advanced Options Pane

Select the desired Clip Plane from the bottom of the Advanced Options section. This sets measurement cutoffs, i.e. data behind the plane will not be measured. Note, clip plane orientation may be changed by selecting Flip Normal. Additionally, the clip plane offset is often set to $0 \mathrm{~mm}$.

If desired you may change the scan density to $1: 1$ for fine detailed, $1: 2$ or $1: 4$ for regions where less curvature exists or accuracy requirements are lower (e.g. 80/20 brackets).

Click Start Capture icon, (Figure 15).

Scanning is initiated by pressing the green button on the scan arm controller. You can pause the scan by clicking the green button on the scan arm controller. Pressing the red 
button ends the scan. During the scanning process the Graphics window shows a realtime depiction of the scan data (Figure 17).

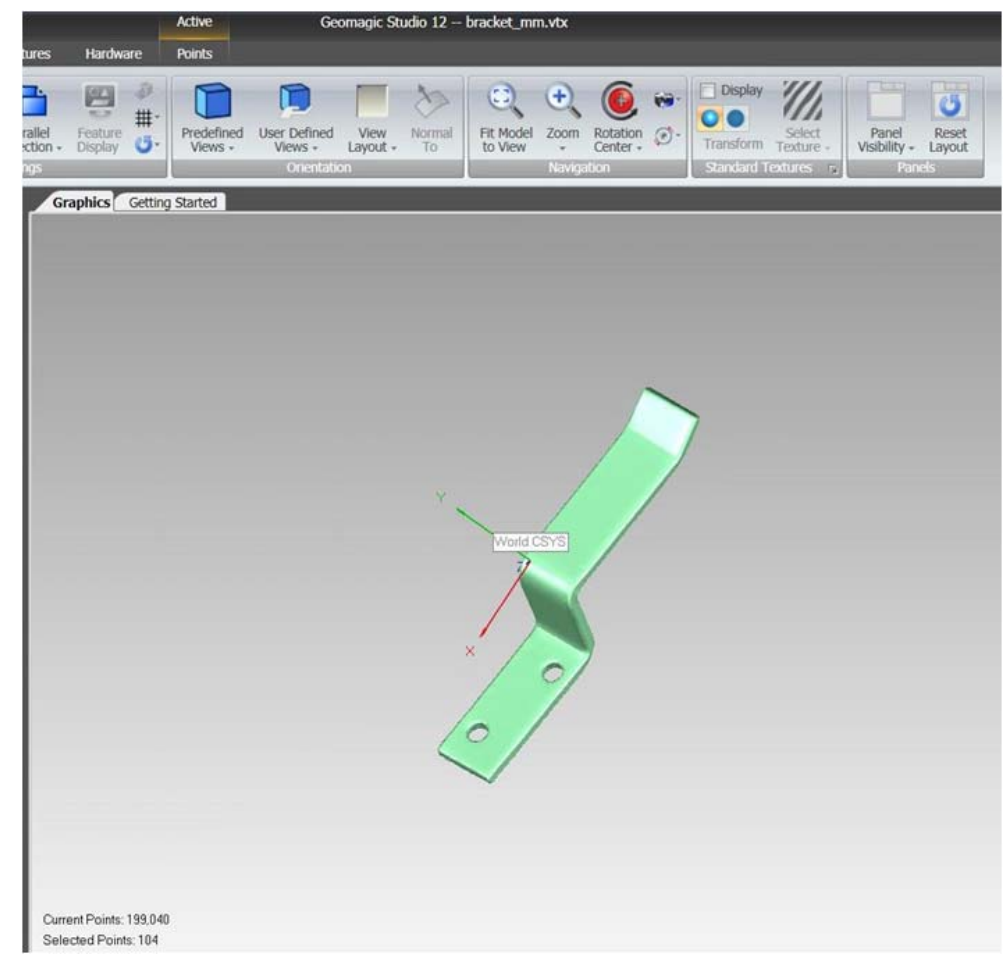

Figure 17. Graphics Display During Laser Scan

The above step can be very time consuming. The model quality needed depends on the application. If the model is to be used for collision detection, then it is not essential that a perfect model is constructed just one that can be used as a representative model.

Note, you may manipulate the view of the sample in the graphics window by clicking and holding the wheel of the mouse.

Note, you may return your view to isometric by pressing the button on the top right of the Graphics pane.

Further details on scanning are available in the GeoMagic studio documentation located in the large three-ring binder labeled "GeoMagic Studio Manual" and on GeoMagic's website. 


\section{Chapter

\section{Refining, Decimating and}

Exporting the Surface Model

Refining and decimating the model is an essential step in the sample model creation process. This process starts with the point cloud generated by the ScanArm then removes outliers in the point cloud data. The number of points in the model is then decreased so that the computer is not over-taxed by its size. First, the multiple scan passes are merged. Then the model is exported into a format recognizable by SScanSS. If the CAD model option is being used then there is no need for this process.

\section{Deleting Outliers/Disconnected Points}

\section{Delete Disconnected}

First we must delete points that are deemed to be disconnected from the model. Under the Points tab click the select disconnected components (Figure 18).

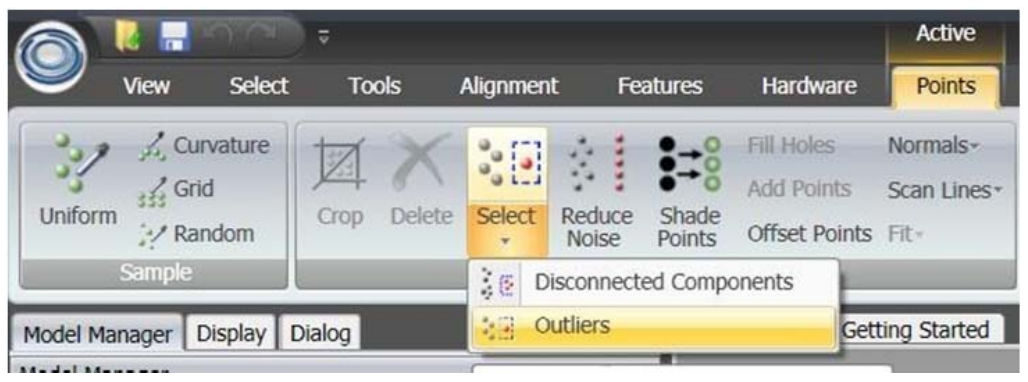

Figure 18. Select Disconnected/Outliers

In Dialog menu, setting separation to medium and the size from 1-3 will select some disconnected points. Click ok and then hit the delete key to delete these disconnected points. 


\section{Select Outliers}

Next click the Select>Outliers (Figure 18). Set the sensitivity to $66.67 \%$ and click OK in the dialog. Hit the delete key to delete the outlier points (should be red).

See GeoMagic Study Manual for other options for improving the point cloud. Knowledge based flow charts are available on the Geomagic website.

\section{Wrap or create surface model from point cloud.}

Highlight desired group in features window > Points tab > Wrap. GeoMagic will open the wrap menu on the left side of your screen (Figure 19).

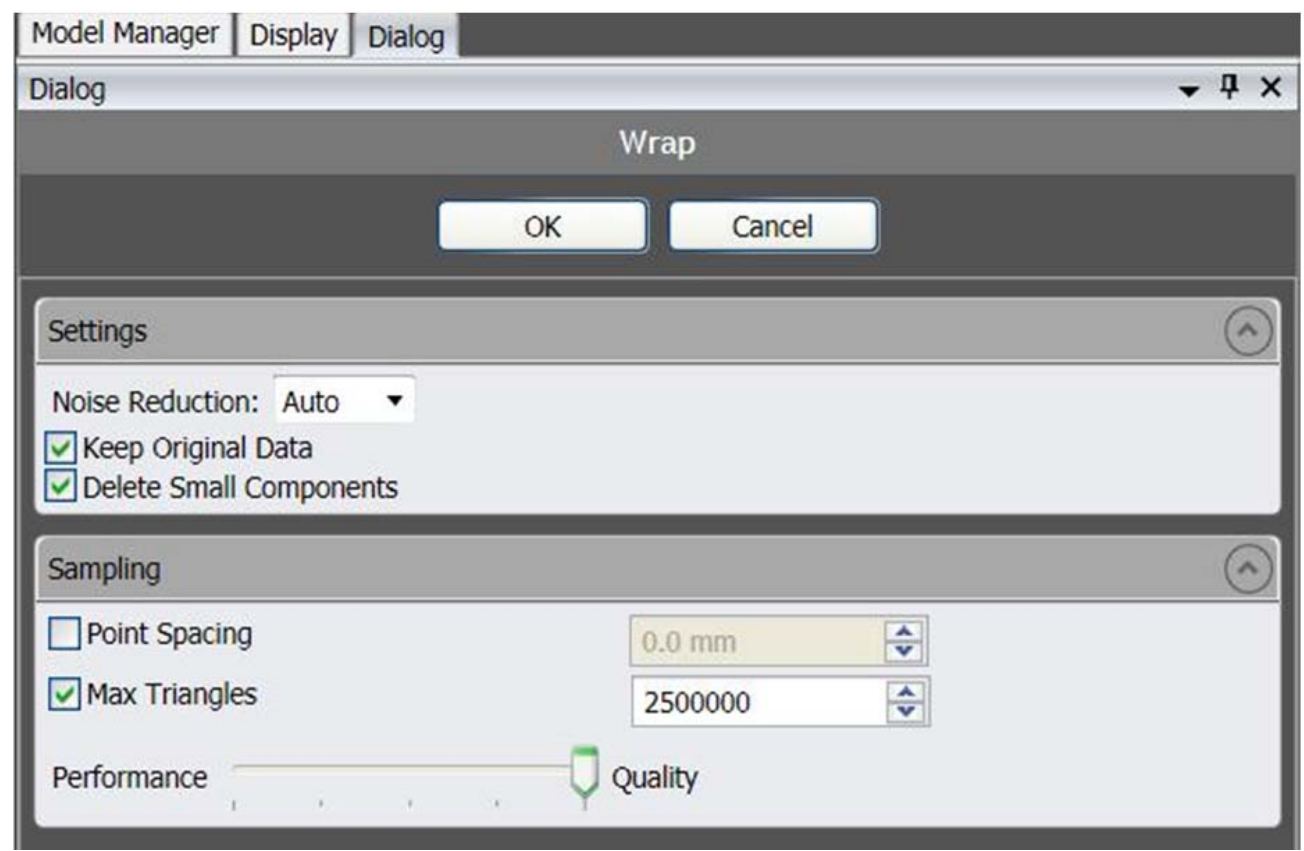

Figure 19. Wrap Dialog 
Note: During Wrap it may be necessary to click and drag over the model in the graphics pane to select all the measured point. If this is done correctly the model should change from green to red. After Wrap the model should be blue (Figure 20)

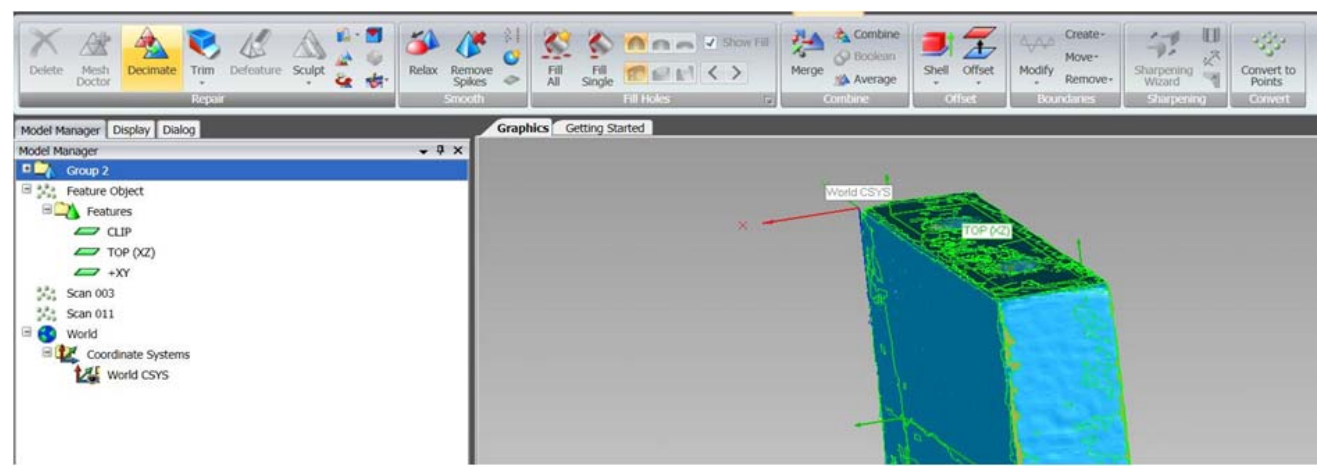

Figure 20. Model After Wrap

Set number of triangles to between 50,000 and 200,000 .

At the Performance-Quality slider bar, move cursor to the quality side of the bar.

Note: Number of triangles may be reduced to save time, but do not go below 150,000 for detailed samples.

Note: These settings may produce a larger model file size but it will give a very accurate representation of the "As is" surface.

Click OK. 


\section{Decimating (Reducing Number of Points/Triangles)}

This step may not be necessary if you selected to remove outliers and reduce noise in the advanced options during the laser scan.

In the polygon menu there is a Decimate button (Figure 21). It is best to define the target number of points to be in the range of 200,000 points (current points are shown in the bottom left of the model window). For large models this may be too few and for smaller models it may be too many. It is possible when collecting the point cloud to increase or decrease the frequency of point collection in different portions of the sample. It is recommended to reduce the collected number of points by at least half. Make needed adjustments and press ok to finish.

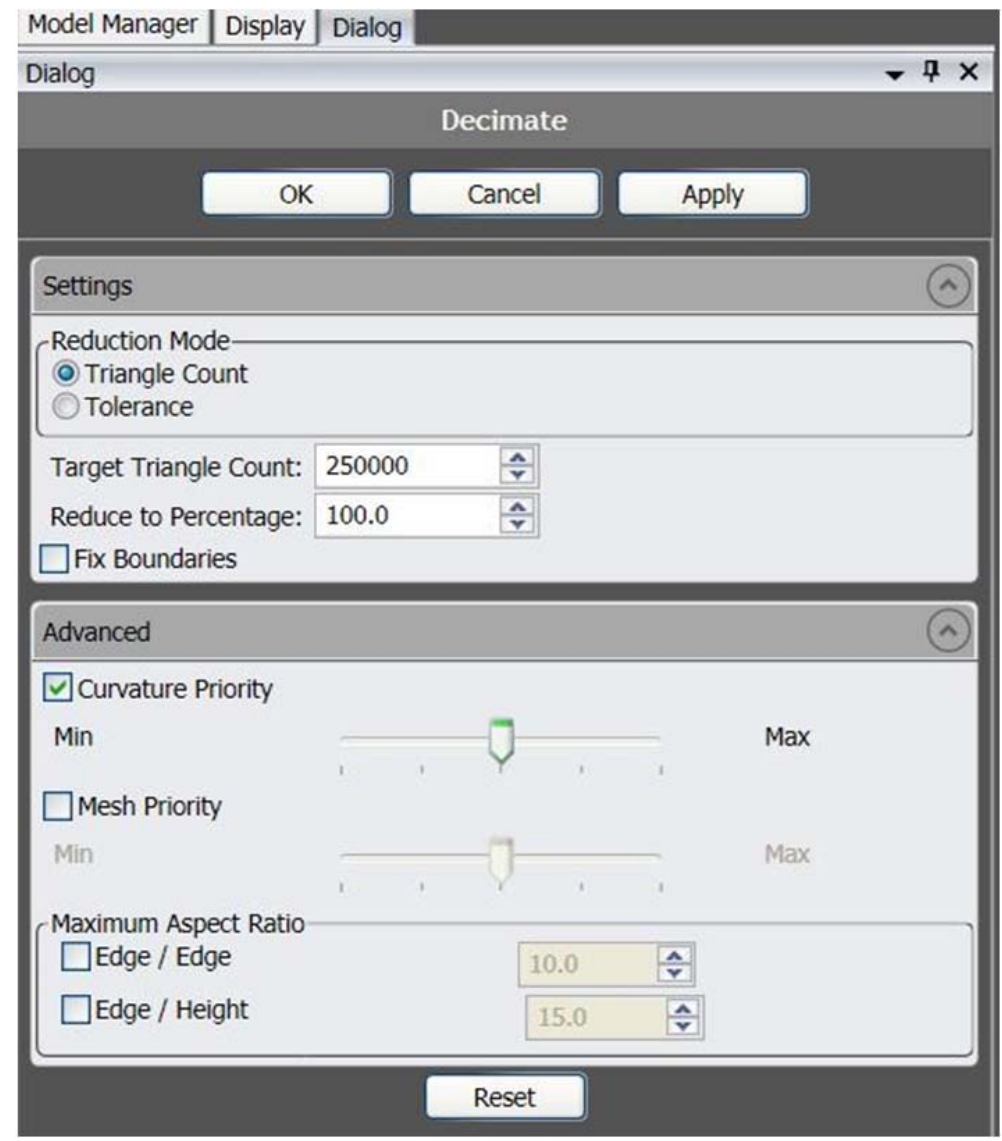

Figure 21. Decimate/Reduce Noise Dialog 


\section{Merging Scans}

Select all individual scans under the Group "\#" folder in the model manager window and go to Points > Merge. Make desired changes and press ok (Figure 22).

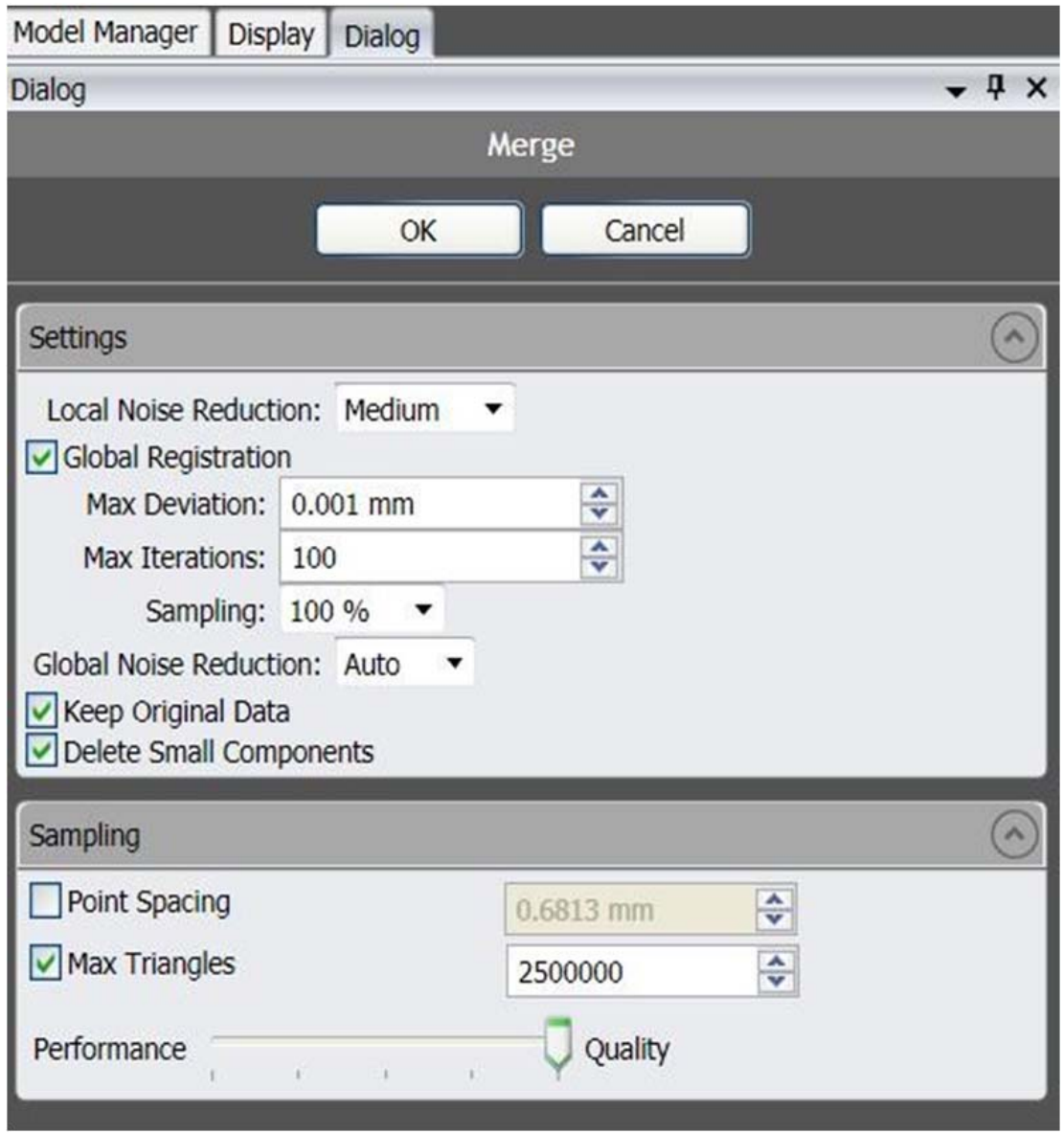

Figure 22. Merge Dialog

Note: In options, make sure Local Noise Reduction is set to "max". The Max Deviation is set at $0.01 \mathrm{~mm}$ and max iterations are set at 100. BE PATIENT. This can take a long time depending on the number of scans and the number of measured points (half an hour or more for large models). 


\section{Exporting the .STL}

After the previous steps are completed, there should be a single surface model of the sample and any attached mounting hardware in GeoMagic Studio graphics pane.

On the left an icon with merged group should have appeared. Right click on the model in the displayed list and select save.

Name the model and change the file type to "STL (ASCII) file (".stl)" then click save.

Next step involves transferring the saved...STL file to the SScanSS computer.

A network drive "ScanArm Share" with IP address 169.254.179.117 has been created so that files may be shared between the GeoMagic and SScanSS computers. Copying the saved file to a flash drive is an alternative transfer option if the local network is not operating. From the 169.254.179.117/ScanArm shared folder on the GeoMagic laptop save the .stl to a flash drive and carry it to the SScanSS computer. 


\section{Craparer

\section{SScansS (In the Offline Alignment Laboratory)}

This chapter details the process by which the instrument simulation program SScanSS is operated. SScanSS will take data collected previously (fiducial point file, measurement file, \& the surface model file) and create a file name.hdf for use at strain measurement instruments. SScanSS is operated twice - once in the offline alignment laboratory and once again after the sample has been mounted on NRSF2 at HFIR or VULCAN at SNS. This chapter is about the SScanSS work that should be done before transportation of the part to one of the instruments.

\section{Starting SScanSS}

Double click the SScanSS icon on the desktop of the offline computer..

Click development.

Click click to continue

Choose ORNL as the facility in the pull-down menu. Choose NRSF2 or VULCAN.

Click login.

If a project folder already exists - click browse and select the folder and click apply. If no folder exists for the project then type a new name in the dialog box and click apply. Hit okay on the dialog box that appears. 


\section{Sample Tab}

On the pull down menu select "import existing model."

On the dialog box that appears (Figure 23), change the filter to ".stl" and select the model file created earlier. The model could either be the "as-is" created surface model or the CAD model, converted, if necessary, to a stl type CAD file.

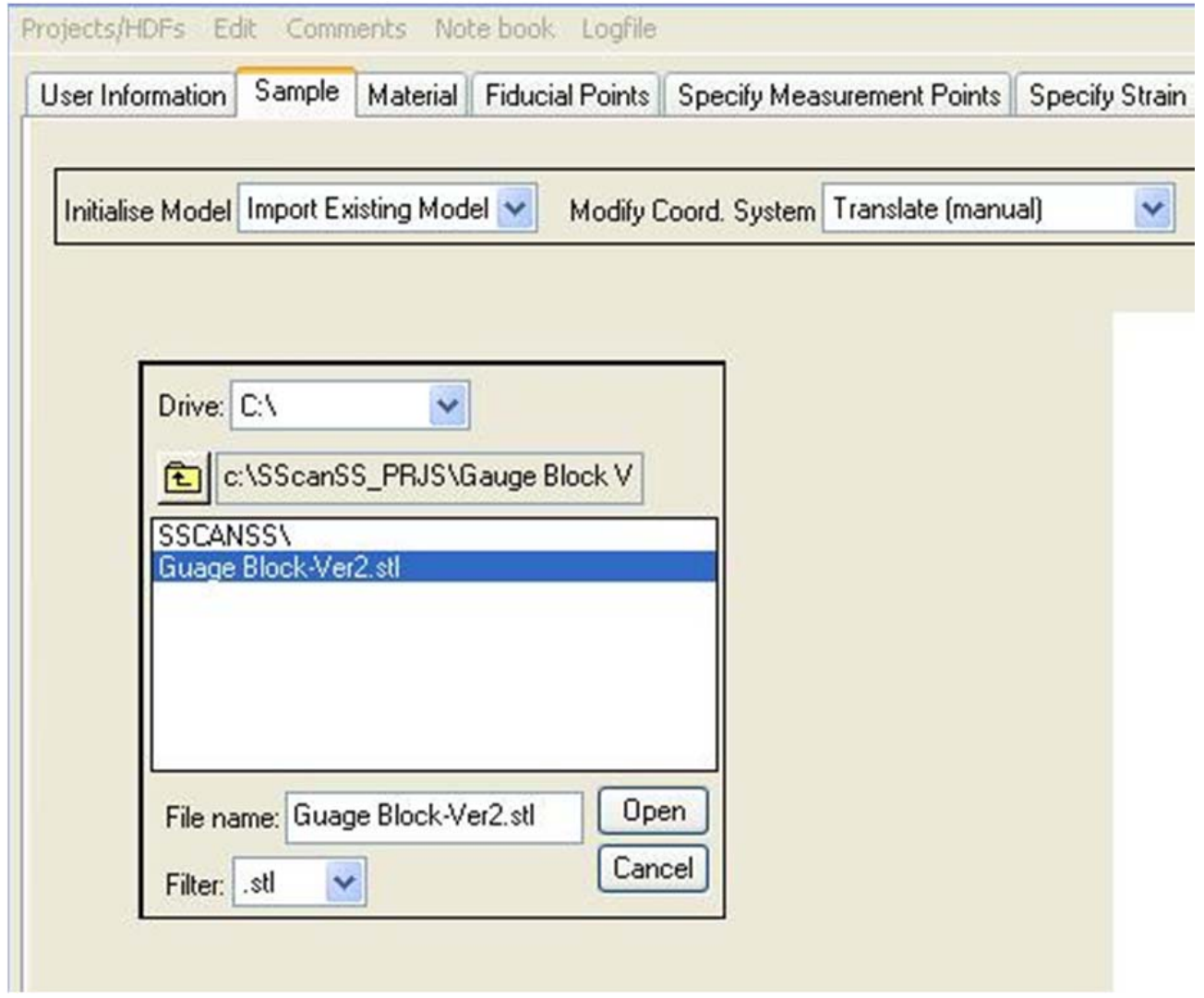

Figure 23. SScanSS .stl Selection Pane

Click yes when asked if you want to convert, and click yes again to denote that the file is an ASCII .stl type CAD file. SScanSS will now create a .model file to be used in the simulation and alignment process. BE PAITENT as this process can be long depending on the size of the model. When asked if you want to find and delete duplicate vertices click no. This process can be used to reduce the size but should have been eliminated by refinement in GeoMagic via merge, decimate, etc. 


\section{Fiducial Points Tab}

On the pull down menu select "import fiducial points." (Figure 24)

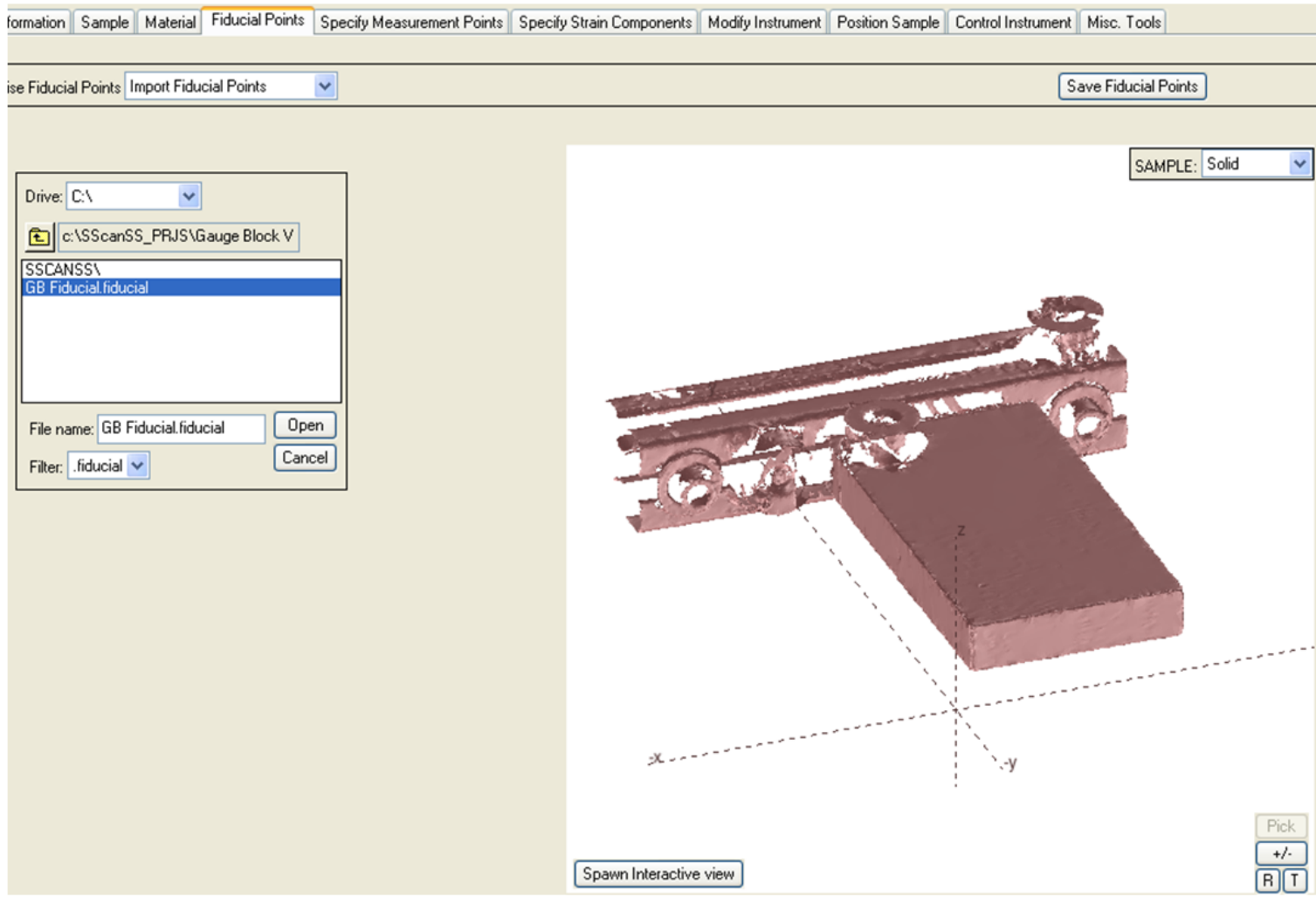

Figure 24. Import Fiducials

Select the .fiducial file created earlier for the model. 
Visually check the screen image of the model and fiducial points to be sure that the points seem reasonable. Use rotate, translate, and zoom options as needed (Figure 25).

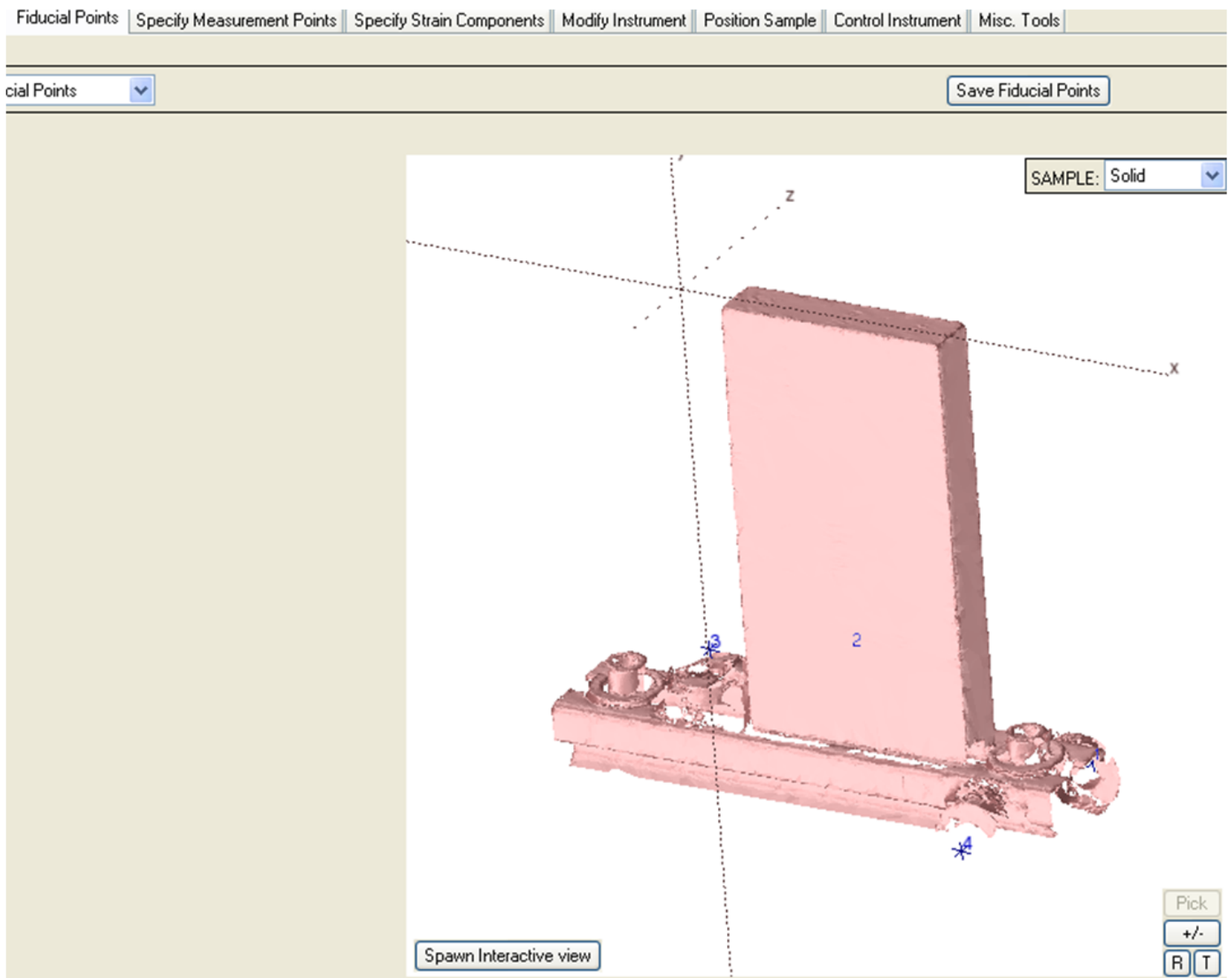

Figure 25. Fiducials Imported

\section{Specify Measurement Points Tab}

A measurement file needs to be created if it has not been already. An Excel process is detailed in Chapter 4 of this document. Select the "import measurement points from file" option in the pull down menu and select the measurement file created for the sample.

SScanSS itself has other tools for defining measurements locations. This approach is recommended for most samples, particularly if any distortion from ideal exists. First, select "manually specify measurement locations" from the pull down menu (Figure 26). Then points can be manually specified by entering their $X, Y$, and $Z$ coordinates in the SCS. Best however, is to use the graphical method that captures the "as-is" features. 


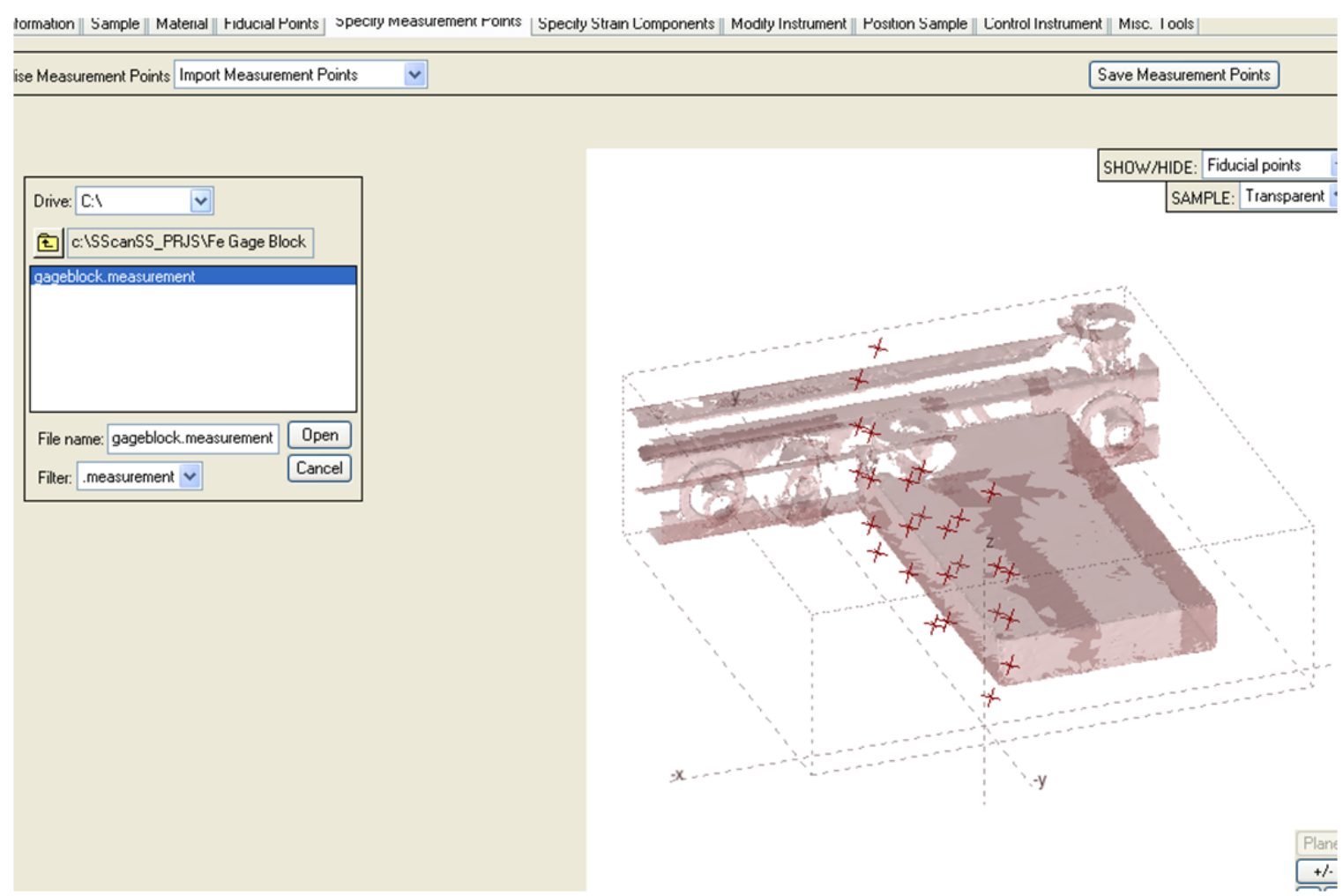

Figure 26. Specify Measurement Points

Choose "graphical" and then choose "cut plane 1' and select the measurement locations using the graphical tools. If a second cut plane is desired, choose and "append" to the previously selected locations. It is often useful to have one of the selected measurement locations at the sample surface. These will be useful in confirming the sample set up after moving sample to the instrument.

Other options exist in the pull down menu as well. These are easily explored and implemented if needed.

\section{Specify Strain Components Tab}

Select "create new measurement vectors" from the pull down menu and using the tools on the left create a vector that is parallel to a direction of the desired strain component. Remember that the vector created will necessarily be aligned parallel with the scattering vector.

\section{Modify Instrument Tab}

In the pull down menu on the left, select NRSF2's optional accessories. For the $Z$ stage the default mounting location is provided, but you can position the Z-stage to various locations on the $\mathrm{XY}$ table and even rotate it to match an alternate mounting. 
In the add/remove hardware pull down menu select "detector." In this menu select the snout to be used in the experiment. Slit sizes will be entered in a separate step later in the process (Figure 27).

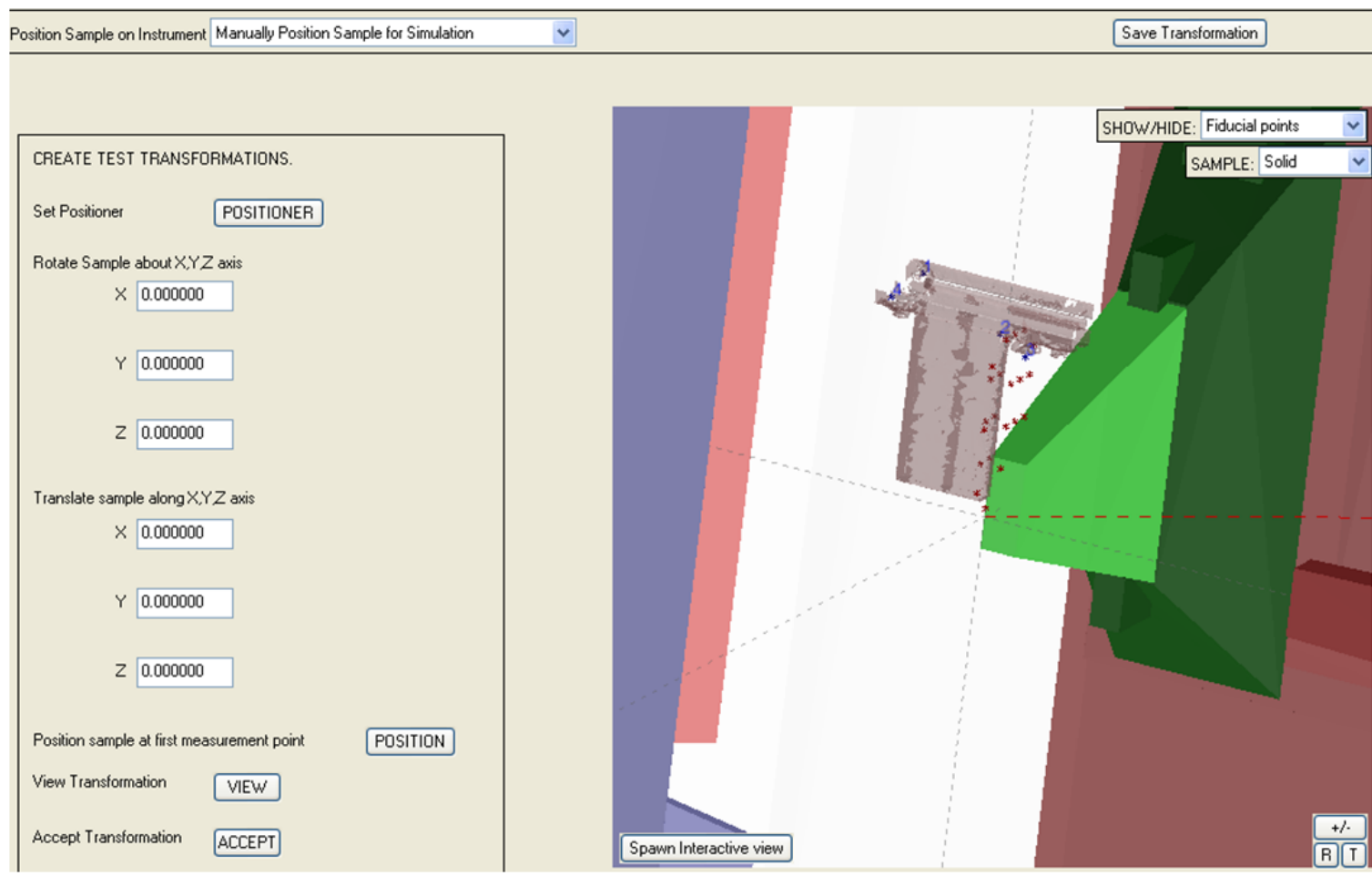

Figure 27. Modify Instrument Pane

Now select "position system" and select whether no Z positioner is needed, or if the Zstage or Z-elevator will be used.

In the reposition movable components pull down menu select the Z-stage if it is being used. Select the "manual translations" option, if not at the default position, corresponding to where the $\mathrm{Z}$ stage is mounted on the $\mathrm{XYZ}$ table. The standard location corresponds to

$$
X=350.00 \quad Y=0.00 \quad Z=0.00 \quad \text { rotation }=90.00 \text {. }
$$

For nonstandard mounting select the "manual rotations about the vertical axis" option and enter the rotation angle.

\section{Saving Progress in a .HDF File}

Save your current progress in SScanSS by clicking the "Projects/HDF" menu on the upper left of the box and clicking "save .hdf on the offline computer. Write to a flash drive or if offline is networked on the ORNL network, copy to the DTP2। HFIR Server with Data FolderlHFIR-4508Transfer. 


\section{Sample Position Simulation (Optional)}

This option may be used for exploring options for how the sample needs to be mounted for selected strain components and to calculate beam path lengths.

Click tab Sample Position. It provides several options such as manual position for simulation and follow prompts. 


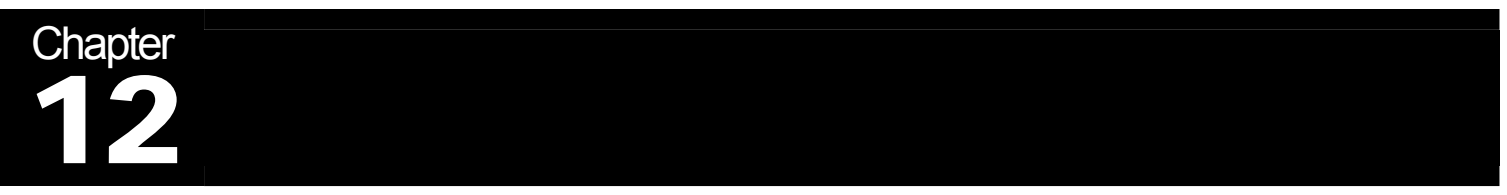

\section{Measuring the Fiducial Points at NRSF2}

Now that experiment planning, sample geometry, and measurement locations are finished and stored in a (name) .hdf file, the sample mount can be moved to a strain measurement instrument. The fiducial points must be measured again in the associated instrument coordinate system. This is achieved by using the laser tracker and CAM2 program. Follow the operational procedures for starting the LaserTracker and CAM2 at the NRSF2 and defining the Sample Positioner System (SPS) coordinate system located in the HFIR beamroom (Appendix B).

\section{Measuring the Fiducial Points Again}

IMPORTANT - FOR EACH FIDUCIAL POINT, RECORD THE $X, Y, Z, A N D \Omega$ OF THE SPS. ONE SETTING OF XYZS IS ALL THAT IS NEEDED, BUT EACH FIDUCIAL CAN BE MEASURED AT ANY SETTING OF XYZ $\Omega$.

Fiducial measurements at HFIR are done with the LaserTracker and CAM2 program. Be sure the tracker in the beam room has been calibrated - ideally check one or two fiducial points in beam room to be sure. The Laser Tracker will self calibrate when CAM2 is opened, do not skip this step unless you have already used the Laser Tracker that same day. Record values to 4 digits after the decimal point and be careful to record the sign as well.

For measuring an SMR in a magnetic nest:

- Click Measure

- Click Point

- Click Comp Off (This deactivates compensation for the radius of the SMR and thus yields the center location of the SMR.)

- Then simply set the SMR into the nest and follow the onscreen instructions to measure the fiducial point 
CAM2 records the measured $X, Y$, and $Z$ coordinates of the center point of the SMR. These can be viewed by selecting "review features."

For fiducial balls (tooling balls) and center punches follow the flowing steps:

- Click Measure

- Click Sphere

Follow screen instruction and measure 10-15 locations about the tooling ball.

- Rotate omega or translate XYZ until ball can be measured using the tracker.

- Use the SMR mounted on the rod with the cup attachment for the fiducial balls. Use the point attachment for the center punch.

- Sweep the rod in a sphere around the fiducial point and measure a large amount of points. Follow the onscreen instructions to finish the procedure.

- CAM2 records the fitted $X, Y$, and $Z$ coordinates of the center of the sphere.

File/Export XYZ data/text - select optics and create file (text)

Export an Excel file with $X Y Z$ values for the fiducial

- Plug in a USB thumb drive

- In CAM2 - select File/Export XYZ Data/Excel - this saves all of the locations determined by the laser tracker in current session

- Use desktop shortcut" Faro Excel" to copy the file to the USB thumb drive. 


\section{Chaper \\ 13}

\section{SScanSS at NRSF2}

Now all the required inputs for alignment have been gathered. The (name) .hdf file contains the fiducial points in the SCS, the measurement locations in the SCS and the model file in the SCS. Now you must input the fiducial point coordinates measured at the instrument in the instrument coordinate system. In this part of SScanSS you will be editing the instrument setup as well as running a virtual scan. The output will be a command file containing the Omega, $X, Y$, and $Z$ for the measurement locations in the Sample Positioning System's coordinate system for each selected location in the measurement file.

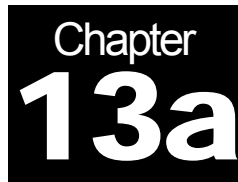

\section{Edit Fiducial Position Excel File}

The computer output to the thumbdrive of the fiducial points and reading them on the instrument computer as it reduces manual input and operator mistyping and the information exists in a file for later reference or modification

Insert USB thumbdrive into NRSF2-BR2 computer and open with Excel. Highlight the rows/columns containing fiducial Pt. \# XYZ for the fiducial points and paste into a new workbook in columns $A, B, C \& D$. Edit column $A$ such that the fiducial point description is just the integer number for the fiducial point.

Add to columns $E, F, G, \& H$ the omega, $X, Y, Z$ corresponding to the particular fiducial point in that row.

Save as a tab delimited text file with extension ".fpos" 


\section{Starting SScansS}

On the NRSF2 - BR2 computer in the beam room, follow the same procedure as detailed in chapter 7.

\section{Position Sample Tab}

On the SScanSS pull down menu select "read measured fiducial point positions from file".

Examine the results data for errors in alignment or fiducial location. If one point has a large error then you may be able to exclude it from the alignment to get a better fit. Accept the fit if it is good enough. Good RMS values are less than 0.08 .

\section{Control Instrument Tab}

Click Detector Control button \& enter values for $2 \theta$, receiving slit offset, and slit size.

Click Jaws Control button \& enter values for incident slit offset and size.

Click Simulate Scan button. Check instant positioner movements and check for collisions (future development) then click run simulation.

If a problem occurs during the virtual scan such as collision detection or if a hardware limit is exceed a dialog box will appear detailing what happened. This could mean a change is needed in sample mounting or in slit offsets.

After the virtual scan is finished click the write script/data/meta-data files and name what the scan will be called and click write script and archive. The Tab file is now created in the SScanSS PRJS folder you created earlier. 


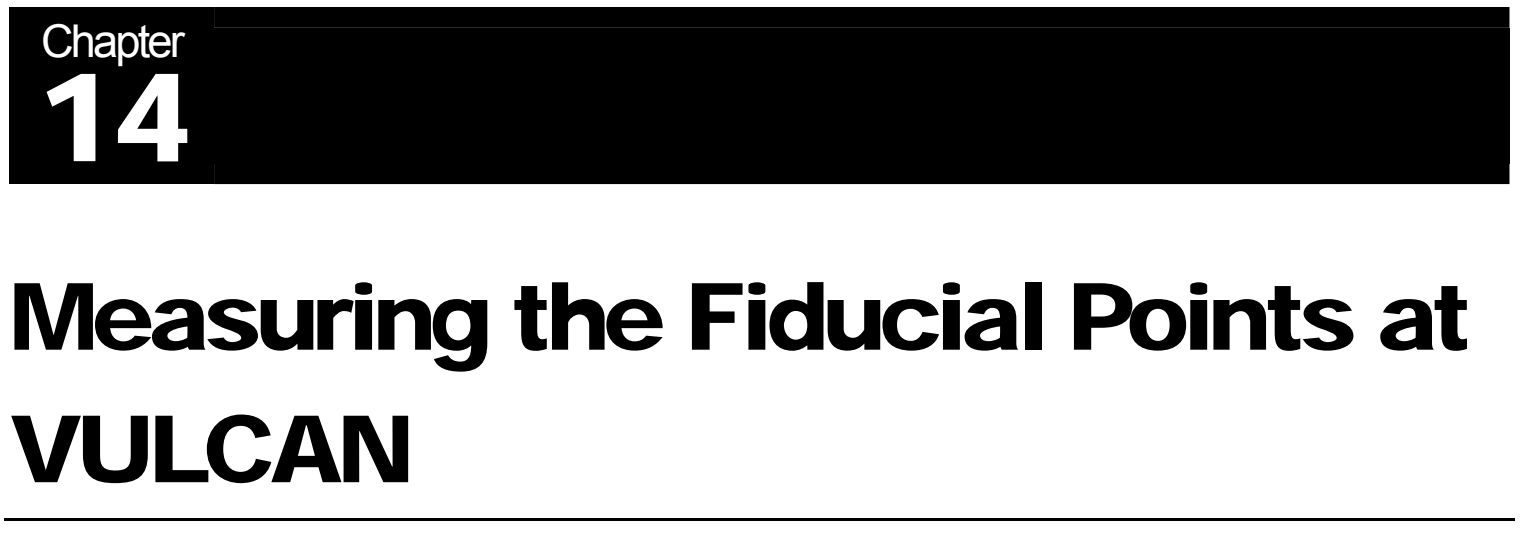

This section of the document is in progress as the VULCAN instrument is still in the early operating phase at SNS. Once it is ready for users who need SScanSS this section will be detailed. 


\section{Chapter \\ 15}

\section{SScanSS at VULCAN}

This section of the document is in progress as the VULCAN instrument is still in the early operation phase at SNS. Once it is ready for users this section will be detailed. 


\section{References}

1. NRSF2 at HFIR: http://www.html.ornl.gov/uc residual.shtml

$$
\text { http://www.ms.ornl.gov/htmlhome/rsuc/rsucmap.html }
$$

Vulcan at SNS: http://neutrons.ornl.gov/instruments/SNS/VULCAN/

2. Hubbard, C.R. "Neutron Diffraction Residual Stress Measurements Within the Phase III Nozzle S-3." Technical Manual, ORNL, TM/2011/191.

3. GeoMagic, 430 Davis Drive, Suite 300, Morrisville, NC 27560; (www.geomagic.com/en/).

4. J.A. James, J.R. Santisteban, L. Edwards and M.R. Daymond, "A Virtual Laboratory for Neutron and Synchrotron Strain Scanning", Physica B, Condensed Matter (2004) 350 pp.743-746.

5. J.A. James and L. Edwards, "Application of robot kinematics methods to the simulation and control of neutron beam line positioning systems", Nuclear Instruments and Methods in Physics Research A. (2007) 571, 709-718.

6. FARO Technologies, Inc., 250 Technology Park, Lake Mary, FL 32746; (www.faro.com).

7. 80/20 Inc., 1701 South 400 East, Columbia City, IN 46725; (http://www.8020.net)). 


\section{Appendix}

\section{Appendix A}

\section{Procedure for Determining NRSF2 Instrument Coordinate System Using FARO Laser Tracker}

1. Set SPS at $x=0.0 y=0.0$

2. Measure $>$ Level (use default name)

3. Measure $>$ Point $>$ CompOff placing an SMR in nest labeled BM_HB2_B_7 on yellow column

a. Use the INSERT key to capture a point and HOME to store

b. Name this point $B H$ POINT

4. Construct $>$ Plane $>$ Parallel.

a. Parallel to level plane through BH Point

b. Label as BH PLANE

5. Measure $>$ Point $>$ CompOff.

a. Point 1: Nest labeled BM_HB2_B_2 is above incident slit system. Name this point TUNNEL

b. Point 2: Nest is on floor behind HB-2B beam stop. Name this point FLOOR

6. Measure $>$ Line $>$ Two Offsets

a. Choose FLOOR and TUNNEL respectively, order is important.

b. Name this BEAM PATH

c. Note BEAM PATH is a line projected onto the $\mathrm{BH}$ plane.

7. Place a nest with $1 / 4$ " shank in one of the outer reamed hole positions on the XYtable.

a. Move omega to -150 degrees.

b. Measure circle in $\mathrm{BH}$ Plane using nest on omega,

c. Moving omega in 60 degree increments from -150 to +150

d. Press HOME at the end of the series to store and name these points

e. Label as OMEGA 
8. Construct 3-2-1 coordinate system.
a. BH PLANE is $+\mathrm{XY}$,
b. BEAM PATH is $+\mathrm{X}$,
c. Origin is OMEGA.
d. Name this NRSF2 COORD SYS

9. Save (.fce) file as NRSF2 COORD SYS cycle-name date (This can be used throughout the entire cycle as long as the Laser Tracker is not moved or bumped.) 


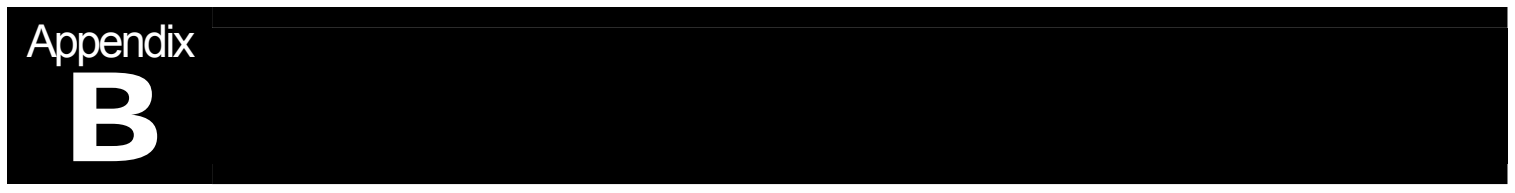

\section{Appendix B}

Quick Reference Guide to Obtaining “As-Is” Model and Exporting Into SScanSS

1. Power up the FARO Scan Arm (Figure B-1)

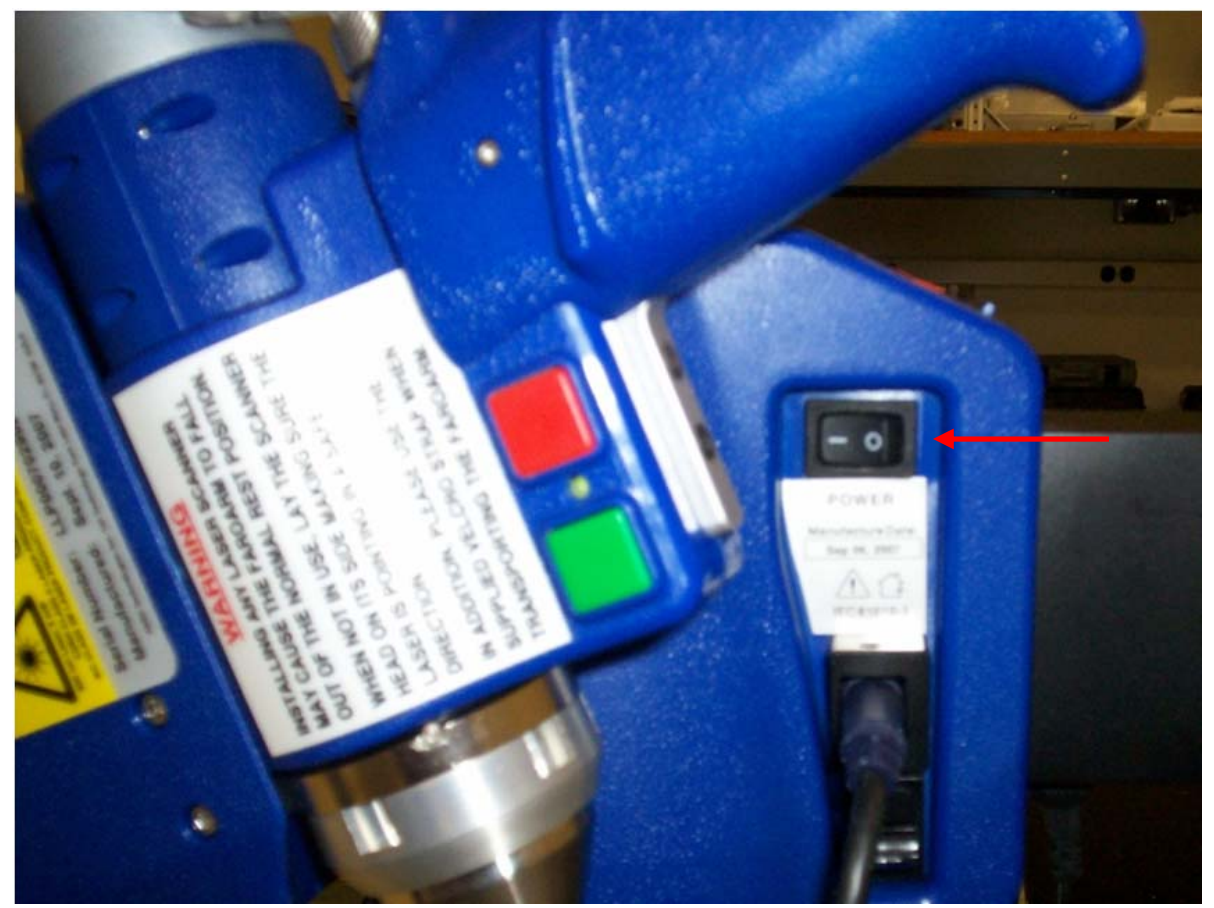

Figure B-1. Scan Arm Power Switch 
2. Start the GeoMagic Studio Software by double clicking the icon (Figure B-2).

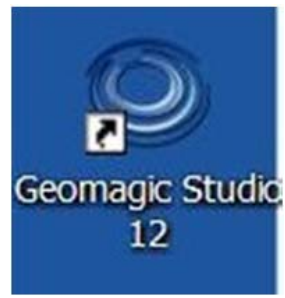

Figure B-2. GeoMagic Desktop Icon

3. Mount Sample to indexer table (Figure B-3) and power on the indexer table via the switch box on the wall by the large monitor.

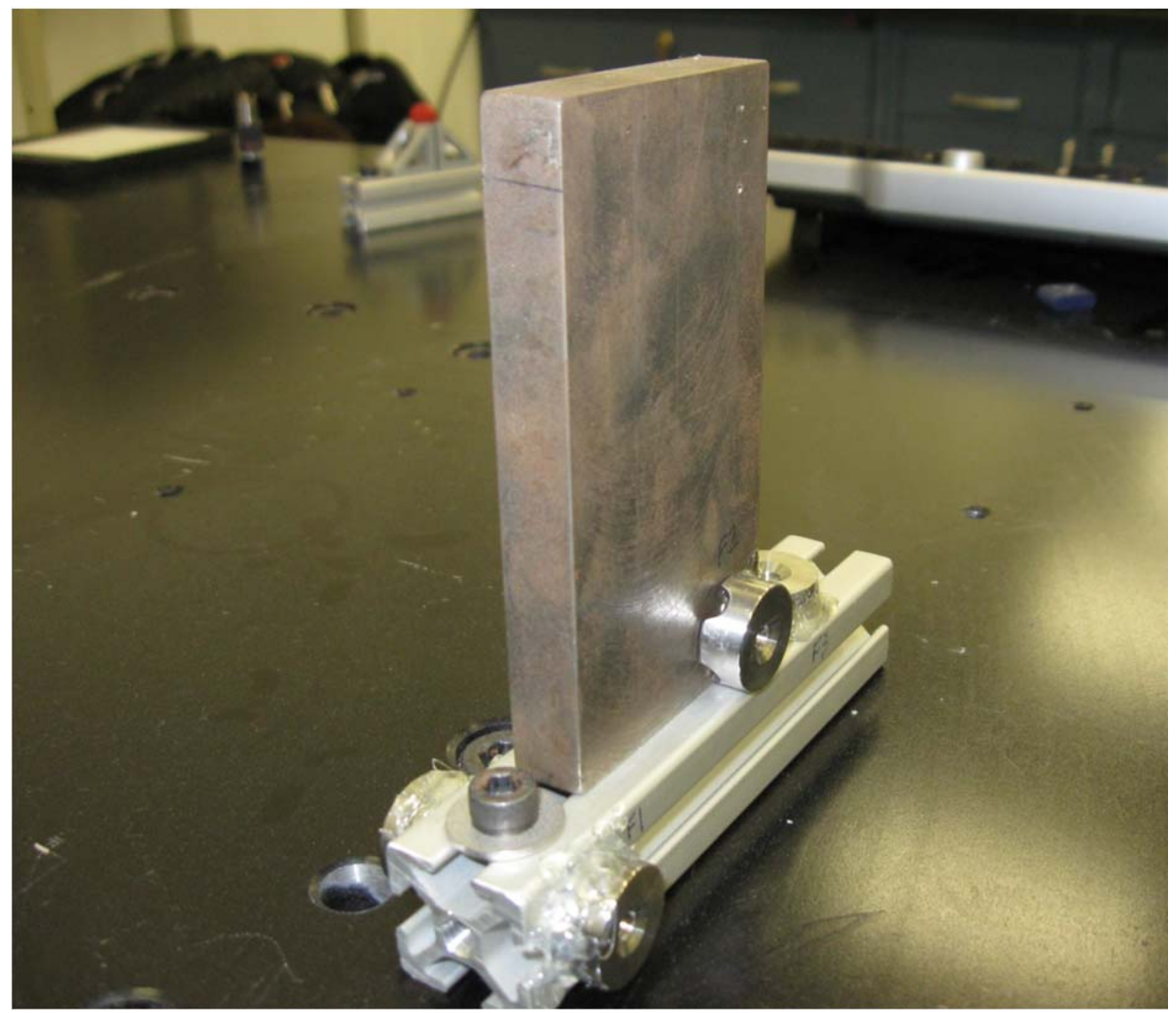

Figure B-3. Sample Mounted to Indexer Table 
4. Mark sample with intended coordinate system and label fiducial nests (Figure B4)

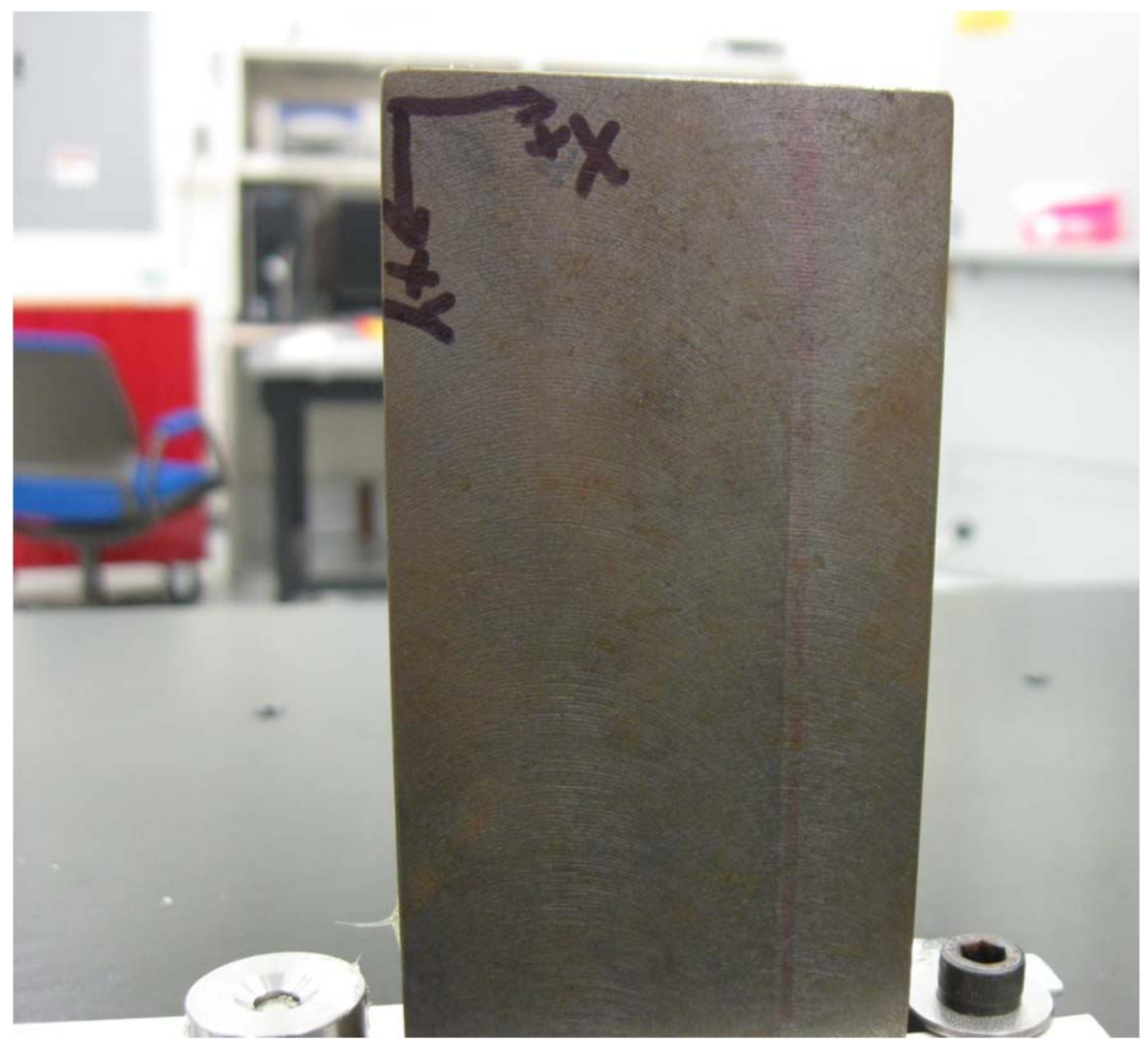

Figure B-4. Sample Labeled With Intended Coordinate System 
5. Go to the Hardware tab in GeoMagic and select the calibrate icon (Figure B-5)

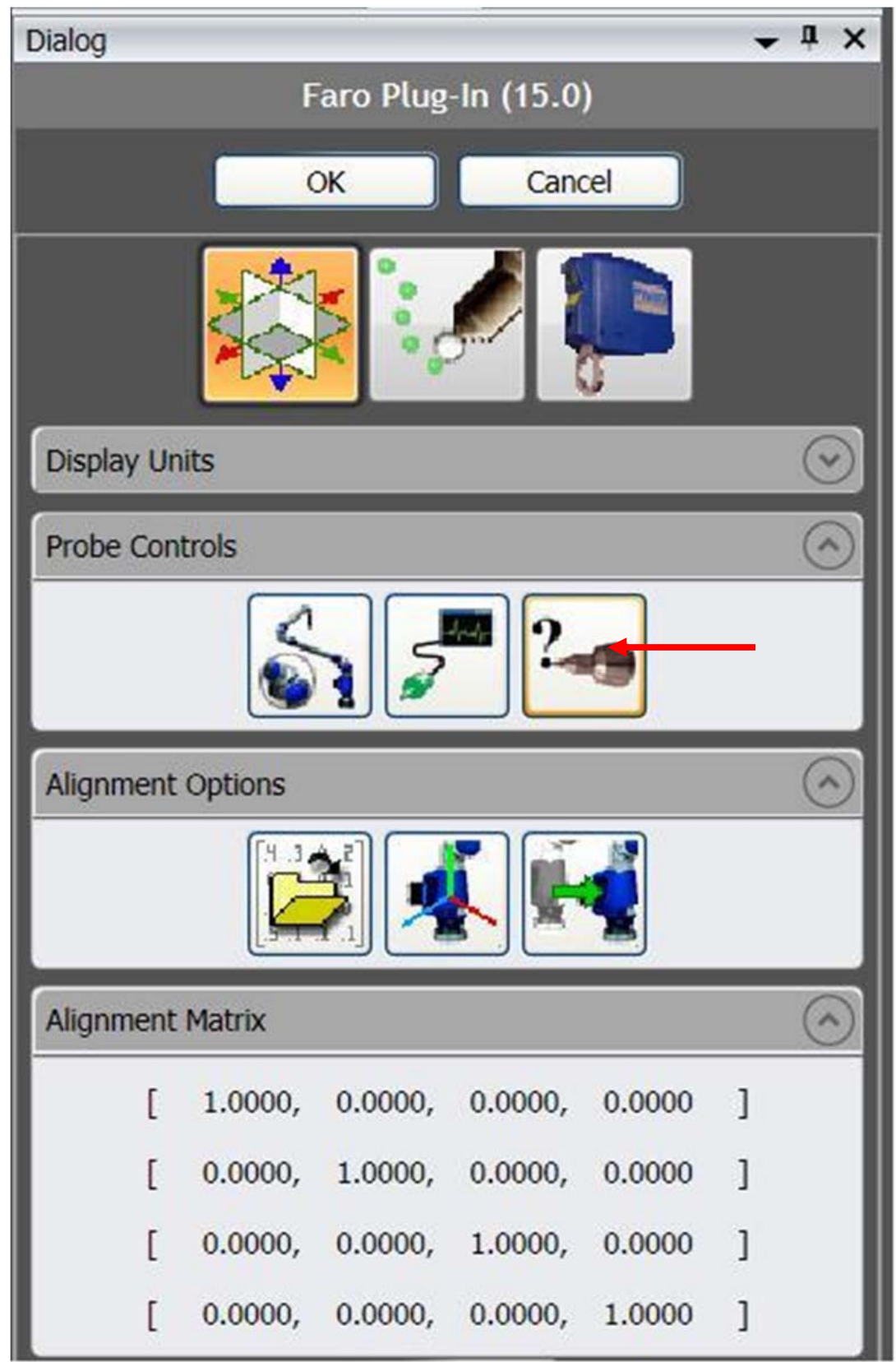

Figure B-5. Hard Probe Option Pane 
6. In the calibration window, select the hard probe hole calibration and calibrate the hard probe followed by a calibration of the laser probe (Figure B-6).

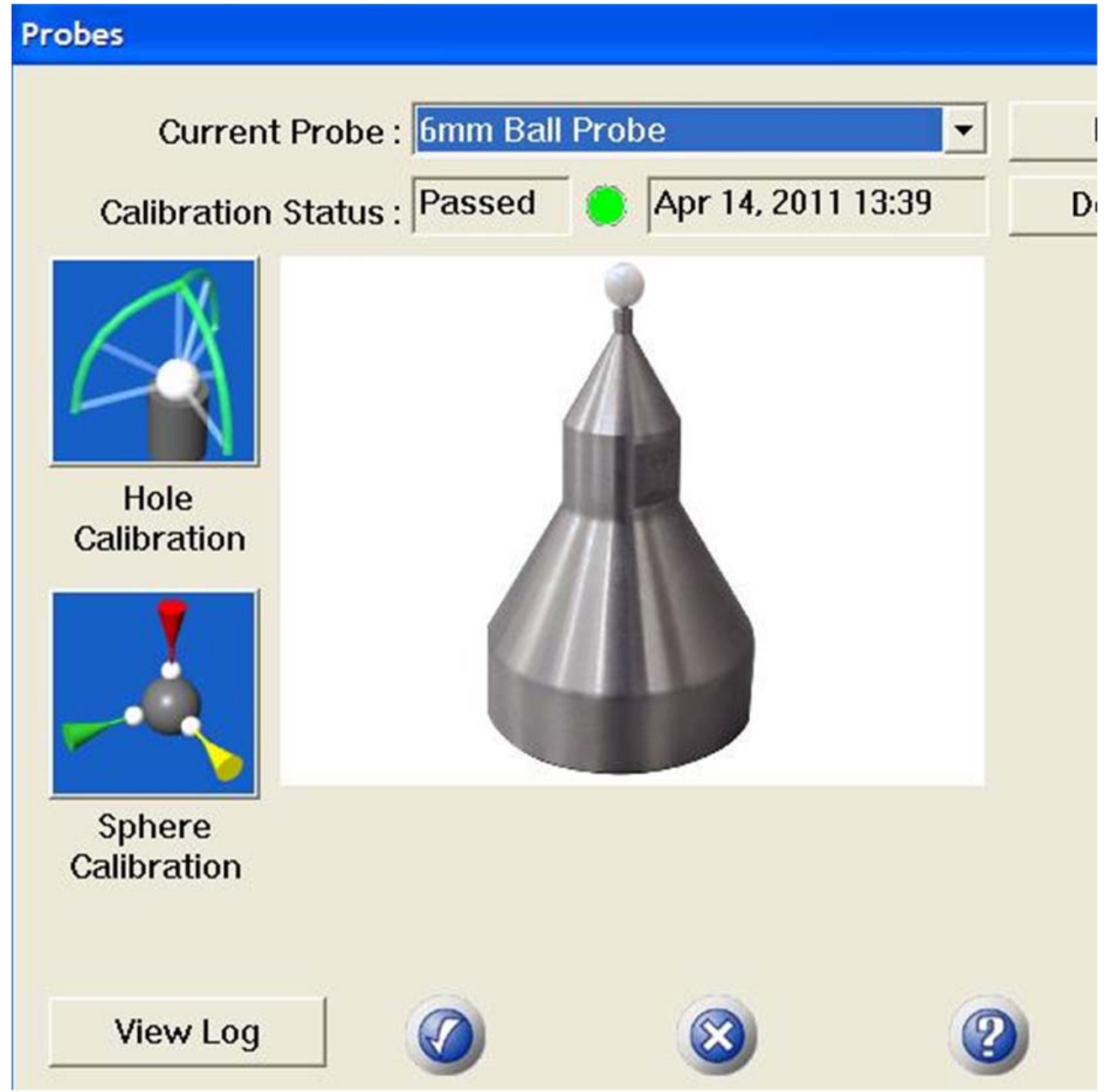

Figure B-6. Calibration Window for Hard Probe and Laser Probe

7. Once the Scan Arm is calibrated it is necessary to define a Clip Plane and coordinate system for the sample inside GeoMagic.

8. Using the Hard Probe capture points along the plane of which you do NOT wish to capture points i.e. the plane of the indexer table, use the red button on the Scan Arm to determine the direction of the plane.

9. Following the Clip plane determine planes along the sample and name them accordingly i.e. "XY plane," along with establishing their respective directions with the red button on the Scan Arm. 
10. Once all planes are captured (at least 3 ) you need to pair them with the world coordinate system to establish a sample coordinate system under the Alignment tab in GeoMagic and clicking the Align to World icon (Figure B-7).

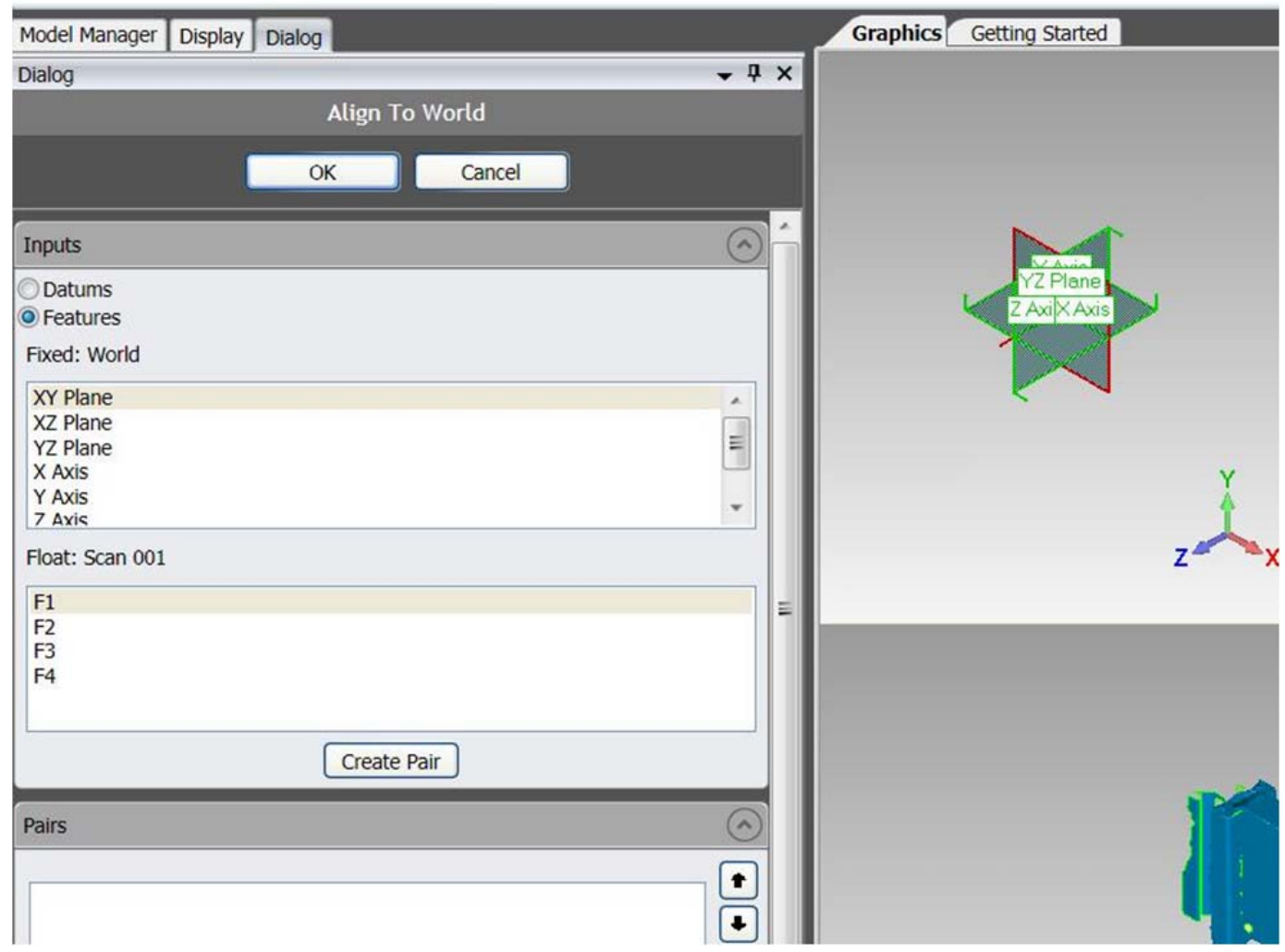

Figure B-7. Align to World Dialog and Pairing 
11. You can see the coordinate origin by going to the View tab and clicking on the show coordinate system icon (Figure B-8).

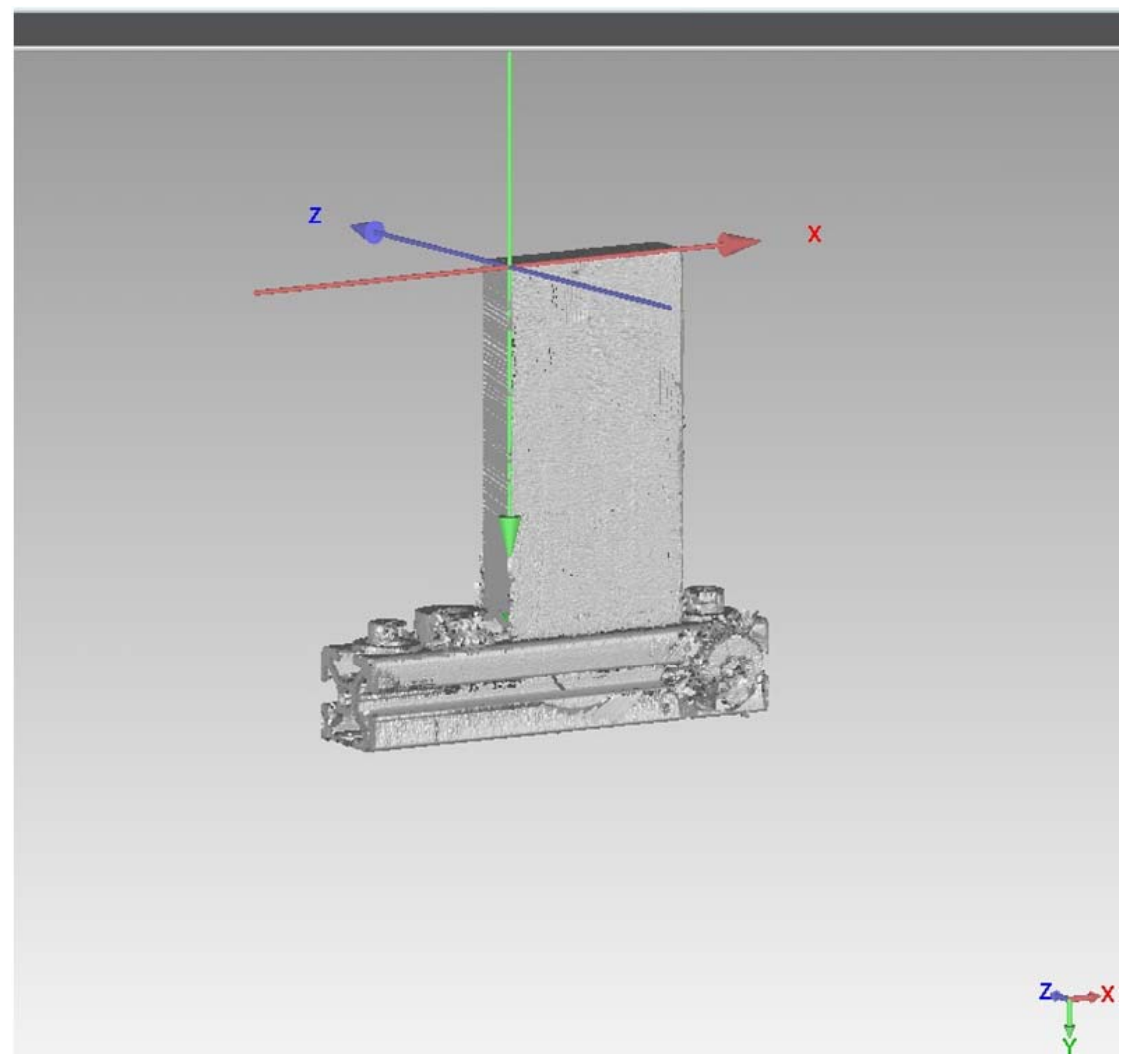

Figure B-8. Show Coordinate System Command 
12. Next capture the fiducial points, which can be done several ways for example, using fiducial spheres in magnetic nest (Figure B-9)

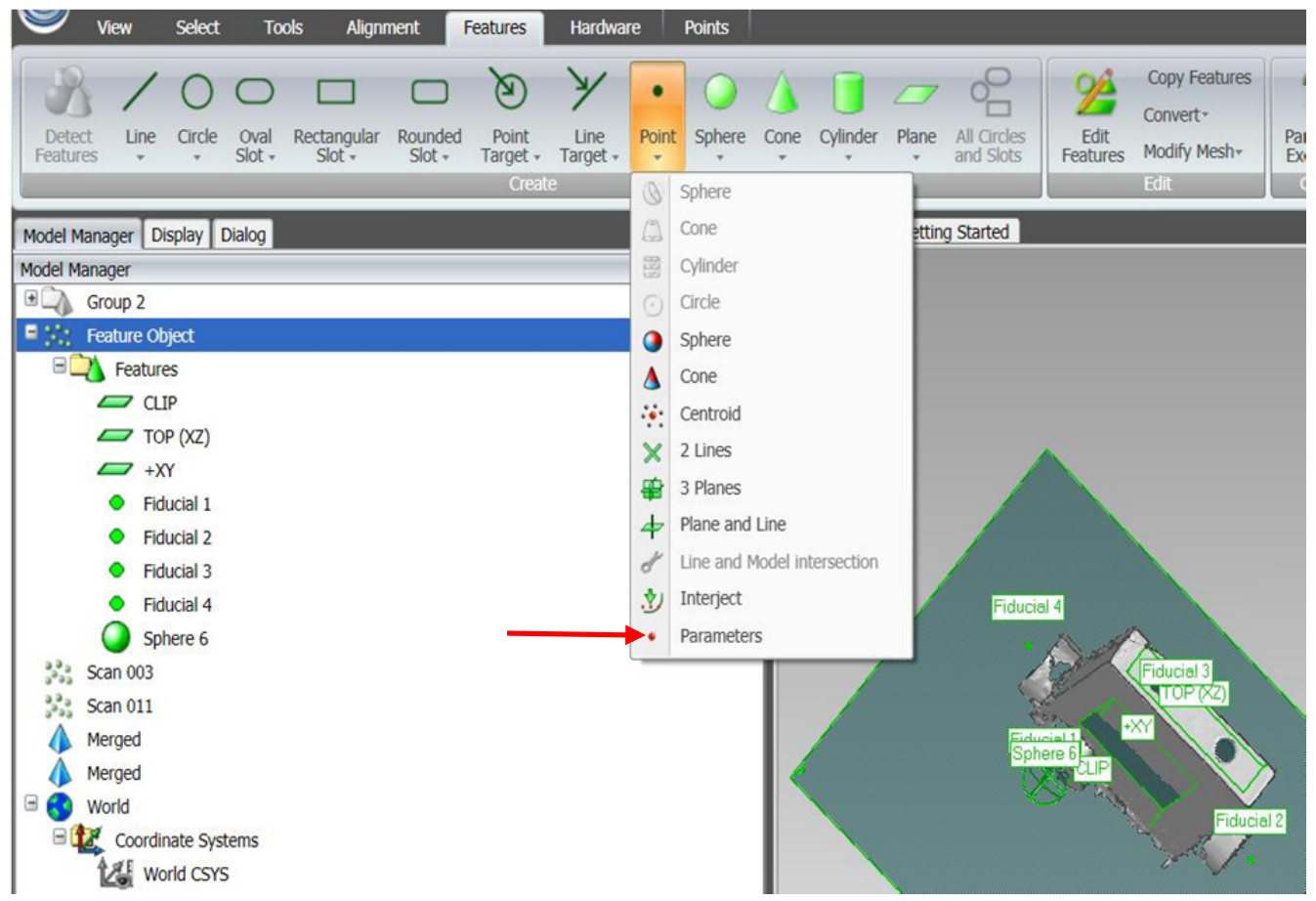

Figure B-9. Fiducial Path

13. Once all spheres have been measured using the hard probe, go to Features tab $>$ Points > Parameters and then select each sphere to convert it to a point and then name that point "Fiducial 1" or "F1" or an appropriate name.

14. Next you will capture the point cloud BUT before doing so, it is necessary to set the scanning medium to an appropriate one for your sample. For shiny, smooth surface use the high scanning parameter and for rough, matte surfaces use the low condition. To access this menu go to Hardware tab > Laser Probe Icon > Scanner Options $>$ then the fourth icon on the right. 
15. Begin to gather the point cloud for the "as-is" surface model using the laser probe capture option under the Hardware tab in GeoMagic (Figure B-10).

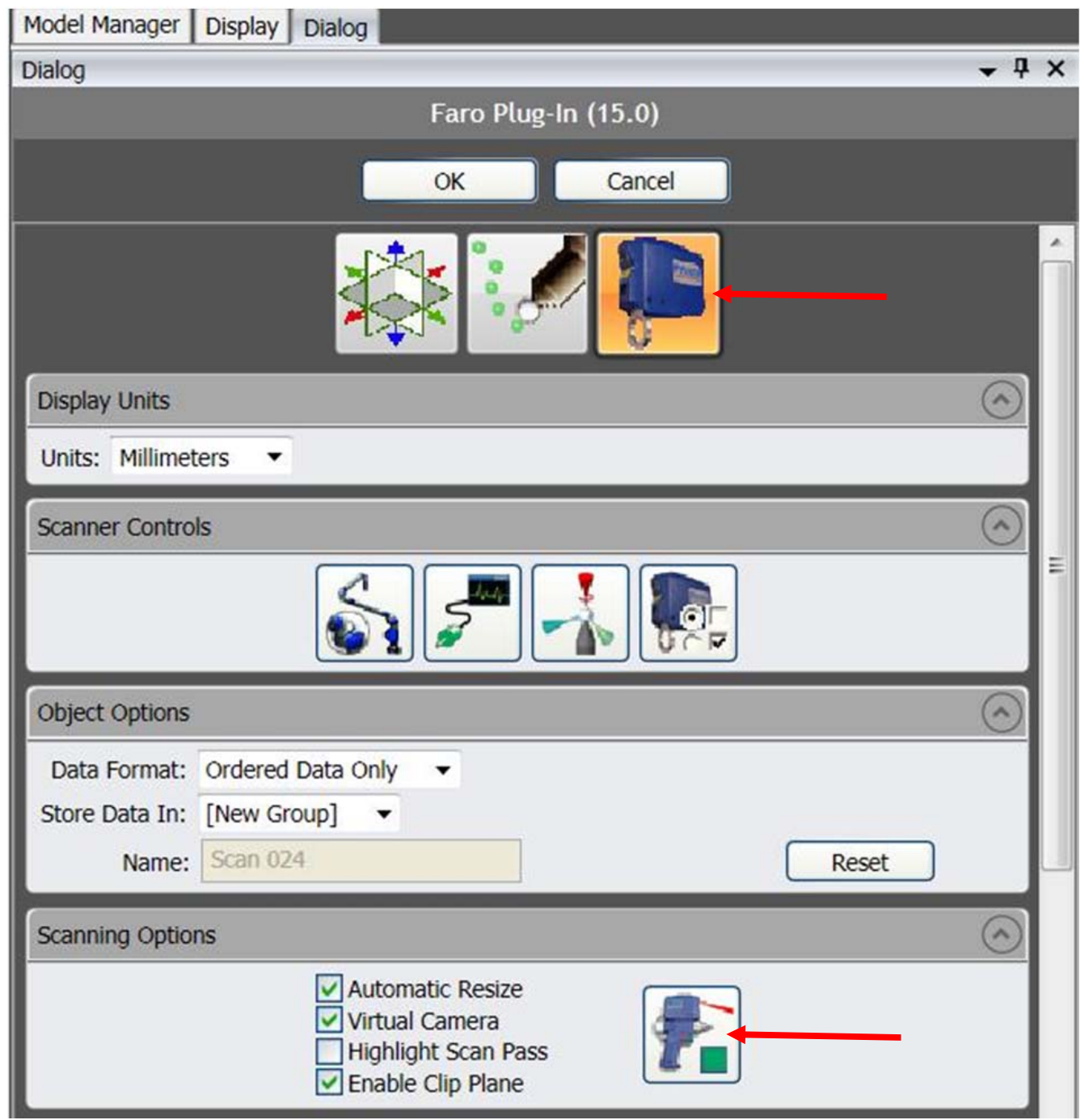

Figure B-10. Laser Probe Dialog Window for Point Capture 
16. You can use the green button on the Scan Arm to pause and reposition yourself for a better scan of the sample. When finished you will see the part rendered as a point cloud in the graphics pane (Figure B-11).

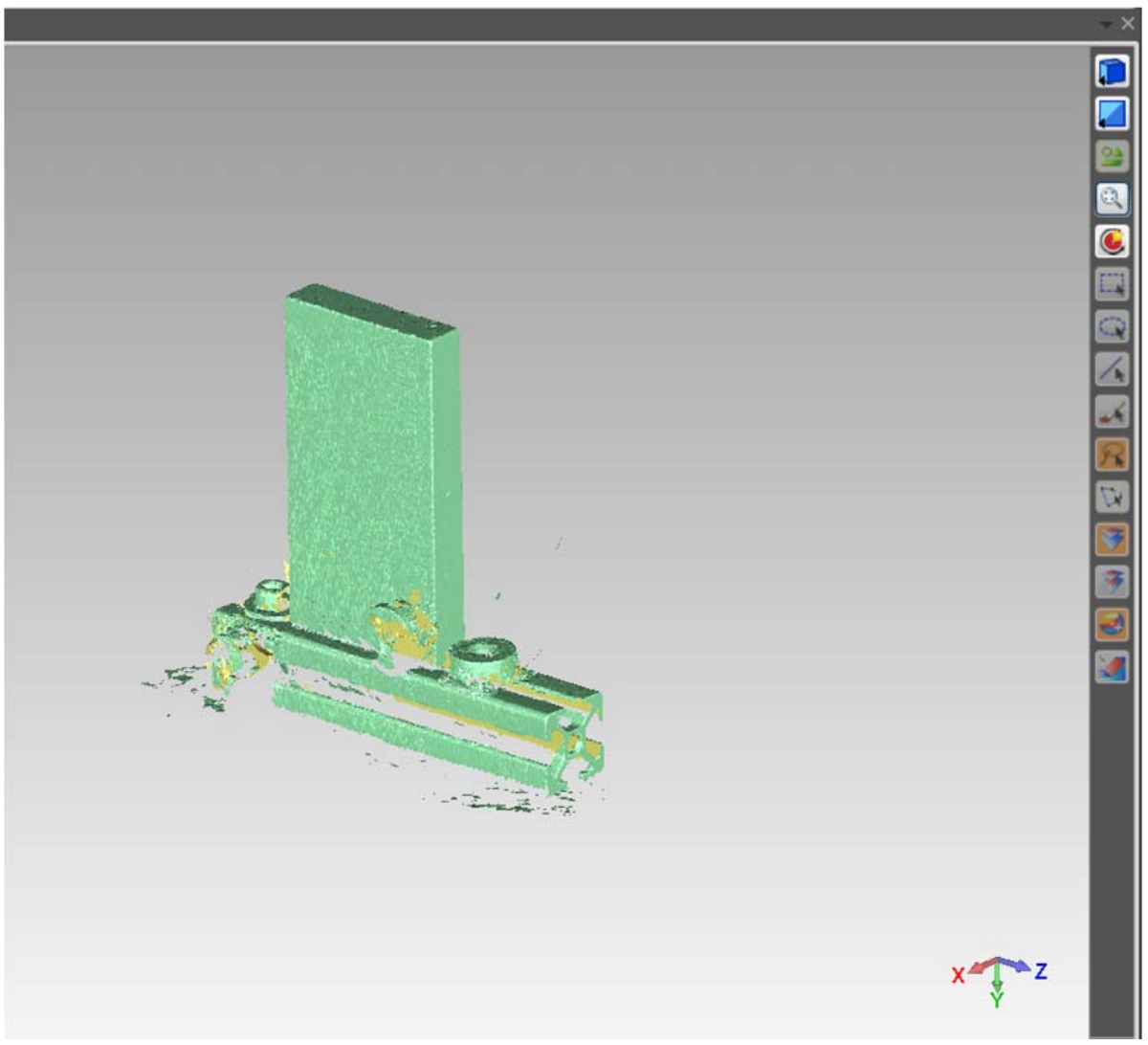

Figure B-11. Point Cloud Rendering of Sample

17. Use the Select Outliers option under the Points tab to select all points that are deemed outside the model (Figure B-12) - you can also select them manually with the lasso tool - selected points will be red and will be deleted upon command execution. 


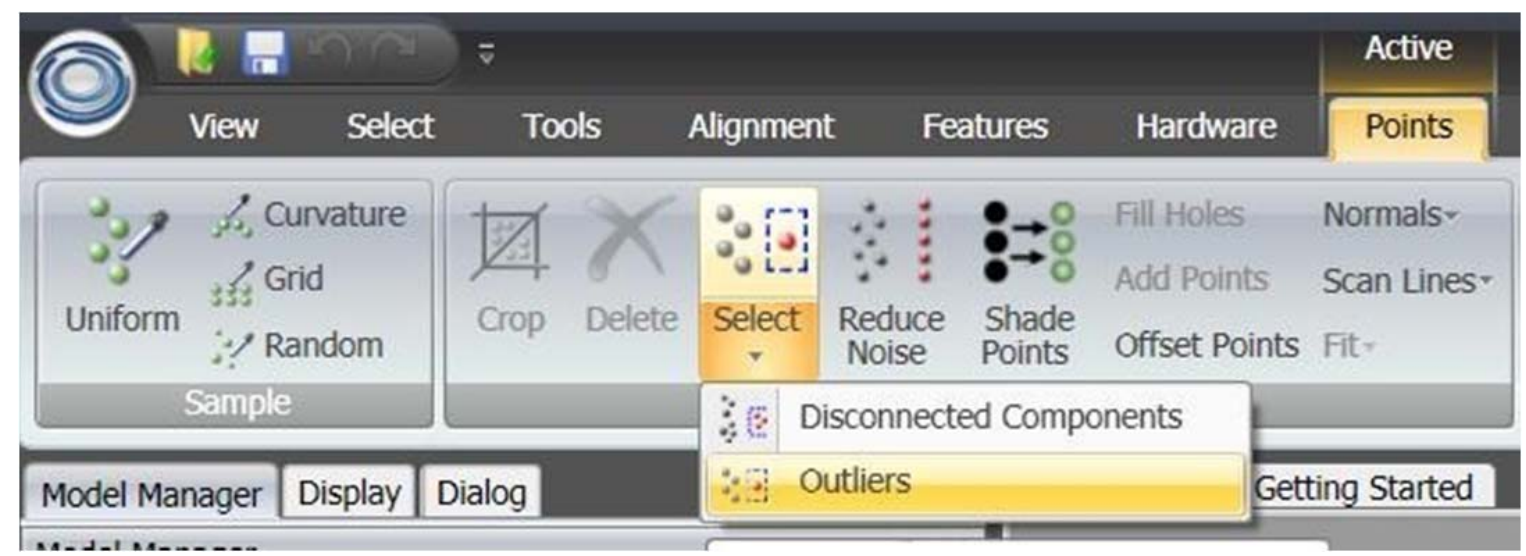

Figure B-12. Select Outliers Command

18. If needed, further refining of the model can be completed using Select Disconnected, Decimate or Wrap under the Points tab which is detailed in chapter 10.

19. Once the model is refined to the desired level it is time to export the stl to the SScanSS software by right clicking on the feature in the Model window and selecting "export." Then save the model to a thumb drive or transfer it to the SScanSS computer in Lab 123 or at NRSF2 at HFIR.

20. Once in SScanSS you must import the existing model (Figure B-13).

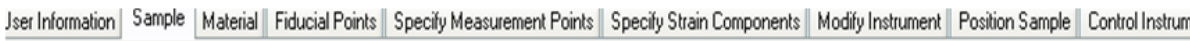

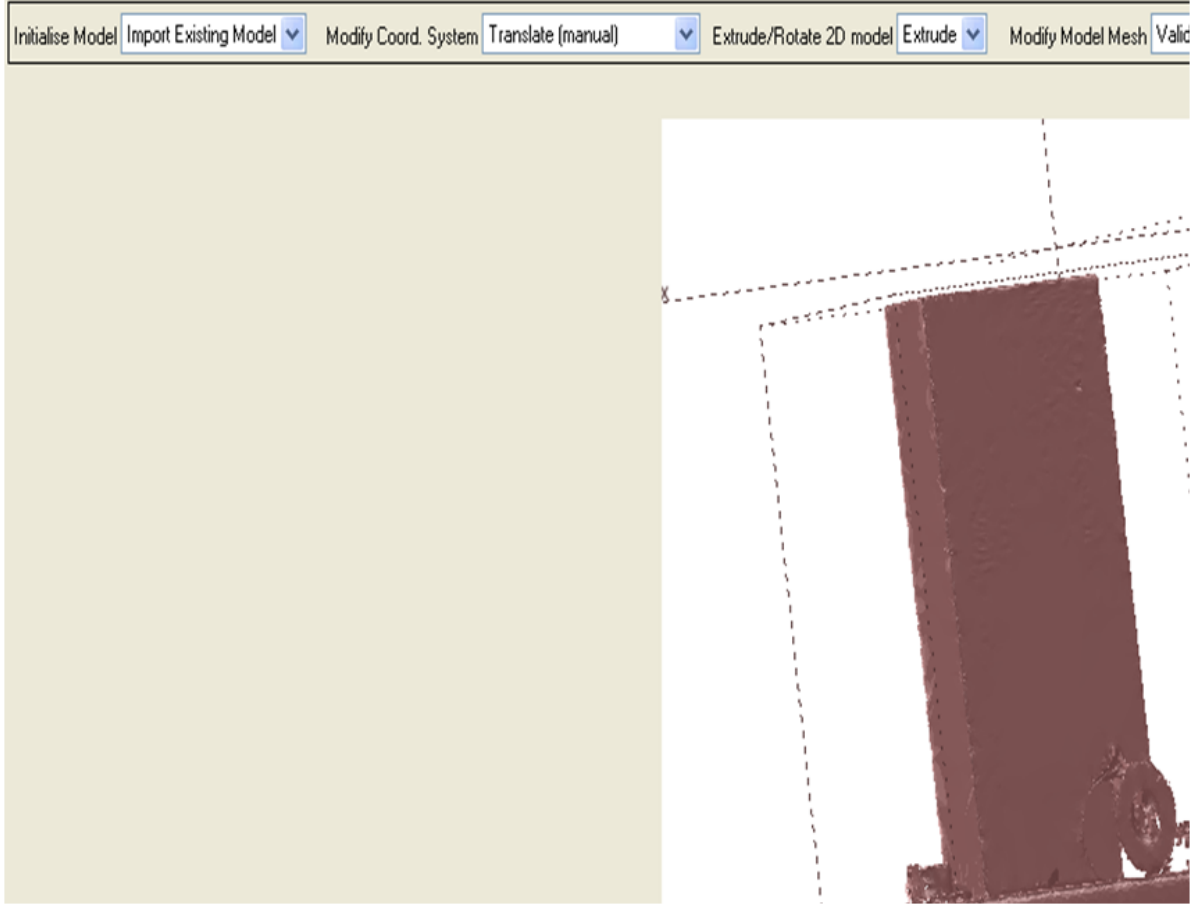

Figure B-13. SScanSS Import Existing Model 
21. Next, import the fiducial points from an excel file or type them in manually (Figure B-14).

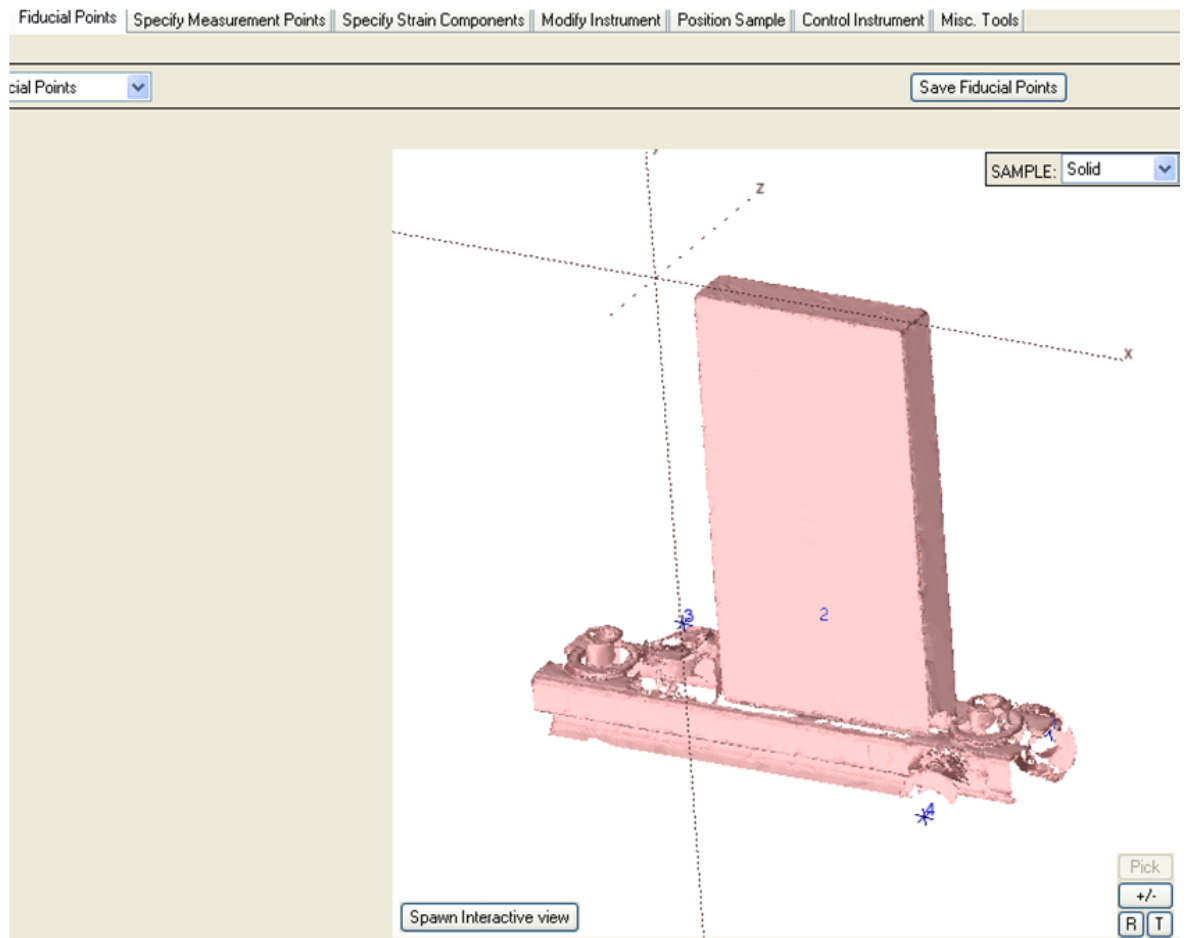

Figure B-14. Importing Fiducials

22. Under the "Specify Measurement Points" you can import or create the measurement points from a file or have SScanSS compute them.

23. From this point you can specify strain components, modify the instrument for use with the Z-Stage or other hardware, reposition the sample to view it inside the instrument, and finally create a tab file for Map software and collecting data. 


\section{Acknowledgements}

Research sponsored by the Assistant Secretary for Energy Efficiency and Renewable Energy, Vehicle Technologies Program, as part of the High Temperature Materials Laboratory User Program, Oak Ridge National Laboratory, managed by UT-Battelle, LLC, for the U.S. Department of Energy under contract number DE-AC05-00OR22725. We would like to acknowledge the exceptional contributions of Mr. William Barton Bailey during the initial phase of this project, involving the LaserTrackers and his contributions to the visualization of NRSF2 within the SScanSS software. We would also like to thank Dr. Jon James, Open University, UK, for al the changes and additions to SScanSS that now make the ORNL Off-Line Alignment and planning for Neutron Diffraction Strain mapping possible. Paris Cornwell, Jeff Bunn and Josh Schmidlin were sponsored by ORISE via the ASTRO Program and Laboratory Technology Program. Also want to thank Brian Cady for his input on the layout and work flow of this manual. 